\title{
Beta seasonal autoregressive moving average models
}

\author{
Fábio M. Bayer* Renato J. Cintra ${ }^{\dagger} \quad$ Francisco Cribari-Neto ${ }^{\ddagger}$
}

\begin{abstract}
In this paper we introduce the class of beta seasonal autoregressive moving average ( $\beta$ SARMA) models for modeling and forecasting time series data that assume values in the standard unit interval. It generalizes the class of beta autoregressive moving average models [Rocha and Cribari-Neto, Test, 2009] by incorporating seasonal dynamics to the model dynamic structure. Besides introducing the new class of models, we develop parameter estimation, hypothesis testing inference, and diagnostic analysis tools. We also discuss out-of-sample forecasting. In particular, we provide closed-form expressions for the conditional score vector and for the conditional Fisher information matrix. We also evaluate the finite sample performances of conditional maximum likelihood estimators and white noise tests using Monte Carlo simulations. An empirical application is presented and discussed.
\end{abstract}

Keywords: Beta ARMA, Beta distribution, Forecasts, Rates and proportions, Seasonal time series, Seasonality.

MSC: 62M10, 62Fxx, 91B84

JEL: C1, C22, C51

\section{Introduction}

Univariate time series modeling is commonly used in many fields. Most conventional time series models are based on the Gaussianity assumption [1]. A well-known class of this linear models is the class of autoregressive integrated moving average models (ARIMA) [2]. However, it has been recognized that the Gaussian assumption is too restrictive for many applications [3]. As a consequence, there has been increased interest in non-Gaussian time series models [4]. Some models for discrete variate time series are considered in [5, 6, 7]. In [8] is proposed a quasi-likelihood approach to regression models for discrete and continuous time series. In [9] is focused on time series modeling under nonGaussian innovations. Non-Gaussian time series models are considered as instantaneous transformations of Gaussian time series in [10,1]. Time series models based on generalized linear models (GLM) [11] are considered in [12, 13 , 14, 15]. Other important and recent works on non-Gaussian time series modeling are [16, 17, 3, 18, 19, 20, 4]. A comprehensive reference on general models for time series analysis is [21].

${ }^{*}$ F. M. Bayer is with the Departamento de Estatística and LACESM, Universidade Federal de Santa Maria, RS, Brazil, e-mail: bayer@ufsm.br

${ }^{\dagger}$ R. J. Cintra is with the Signal Processing Group, Departamento de Estatística, Universidade Federal de Pernambuco, PE, Brazil, e-mail: rjdsc@de.ufpe.br

${ }^{\ddagger}$ F. Cribari-Neto is with the Departamento de Estatística, Universidade Federal de Pernambuco, PE, Brazil, e-mail: cribari@de.ufpe.br 
Practitioners are oftentimes interested in modeling the behavior of variables that assume values in the standard unit interval, $(0,1)$, such as rates and proportions [22, 23, 24, 25, 26, 27, 28, 29]. Time series modeling of such variables can be accomplished by using the class of beta autoregressive moving average models ( $\beta$ ARMA) [30]. It is noteworthy that the beta distribution is quite flexible since its density can assume a variety of different shapes depending on the values of the parameters that index the distribution: it can be symmetric, left-skewed, right-skewed, constant, J-shaped, and inverted J-shaped [24, 30, 31]. According to [31], "there are situations where the response variable is continuous and bounded above and below such as rates, percentages, indexes and proportions. In such situations, the traditional GLMM based on the Gaussian distribution is not adequate, since bounding is ignored. An approach that has been used to model this type of data is based on the beta distribution." Recent related works include [32, 29, 33, 34].

Time series data may exhibit periodical fluctuations, i.e., they may display seasonality. Models that include seasonality have been extensively explored in the literature [35 36, 37], the seasonal ARIMA model (SARIMA) [2] being the most used model for Gaussian seasonal data. A commonly used approach for dealing with non-Gaussian data is to assume that seasonal fluctuations are deterministic and then model them using sine/cosine functions as covariates in regression times series models; see [29, 38, 14]. Such an strategy, however, is not appropriate when the seasonality is driven by a stochastic mechanism [39]. Some authors have recently devoted attention to non-Gaussian seasonal time series models; see, e.g., [39, 40]. To the best of our knowledge, however, no seasonal time series model is available for variables that assume values in the standard unit interval, such as rates and proportions.

Our chief goal is to introduce a time series model based on the beta law that includes stochastic seasonal dynamics: the beta seasonal autoregressive moving average model ( $\beta$ SARMA). We also outline maximum likelihood parameter estimation, obtain closed-form expressions for the conditional score function and for the conditional Fisher information matrix, show how confidence intervals can be constructed and how hypothesis testing inference can be performed (including a seasonality test), address the issue of model selection, propose different residuals that can be used to assess goodness-of-fit, present white noise tests based on such residuals, and show how out-of-sample forecasts can be produced. Additionally, we present results from Monte Carlo simulations that were carried out to evaluate the accuracy of maximum likelihood estimation and white noise testing inference in finite samples. Finally, we present and discuss an empirical application. We note that the proposed $\beta$ SARMA finds potential applications in a plethora of scientific areas, such as mortality rate [41], seasonal infectious disease [42 43] 44], unemployment rate [34] 30], seasonal variation of fixed and volatile oil percentage [45], solar radiation [46], periodic ocean waves [47], and also in hydrological applications [48, 49].

The paper unfolds as follows. Section 2 introduces the seasonal beta autoregressive moving average model. Several particular cases of the proposed model are examined. Parameter estimation via conditional maximum likelihood is outlined in Section 3 We provide closed-form expressions for the first derivatives of conditional log-likelihood function (score function) and for the conditional information matrix. Interval estimation and hypothesis testing strate- 


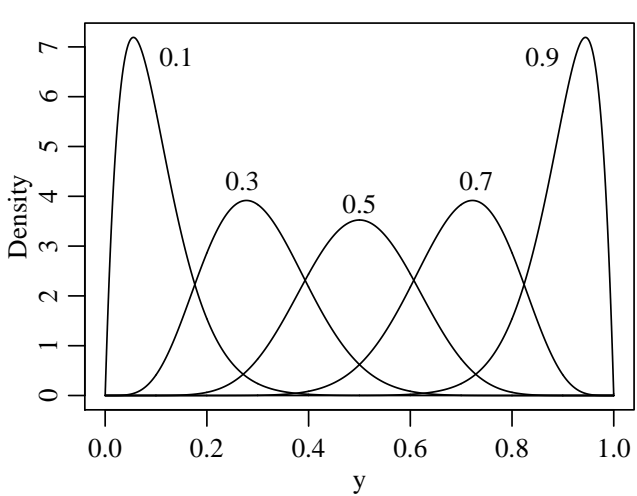

(a) $\varphi=20$

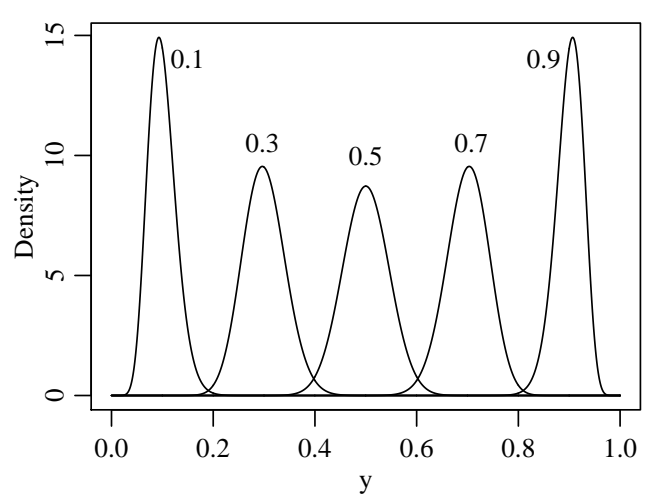

(b) $\varphi=120$

Figure 1: Beta density functions: $\mu=0.10,0.30,0.50,0.70,0.90$ and two values of $\varphi$.

gies are also presented. Section 4 addresses model selection, residuals, and diagnostic analysis. Section 5 contains Monte Carlo simulation results on parameter estimation and white noise testing. An empirical application is presented and discussed in Section 6 Finally, Section 7 offers some concluding remarks.

\section{The proposed model}

Let $\boldsymbol{y}=\left(y_{1}, \ldots, y_{n}\right)^{\top}$ be a vector of $n$ random variables, each $y_{t}, t=1,2, \ldots, n$, being beta distributed conditional on the set of previous information $\mathscr{F}_{t-1}$. The distribution parameters are $\mu_{t}$ (mean) and $\varphi$ (precision). The conditional density of $y_{t}$ given $\mathscr{F}_{t-1}$ is

$$
f\left(y_{t} \mid \mathscr{F}_{t-1}\right)=\frac{\Gamma(\varphi)}{\Gamma\left(\mu_{t} \varphi\right) \Gamma\left(\left(1-\mu_{t}\right) \varphi\right)} y_{t}^{\mu_{t} \varphi-1}\left(1-y_{t}\right)^{\left(1-\mu_{t}\right) \varphi-1}, \quad 0<y_{t}<1
$$

where $0<\mu_{t}<1$ and $\varphi>0$. Figure 1 presents beta densities for different parameter values. The conditional mean and the conditional variance of $y_{t}$ are given by

$$
\begin{aligned}
\mathbb{E}\left(y_{t} \mid \mathscr{F}_{t-1}\right) & =\mu_{t}, \\
\operatorname{Var}\left(y_{t} \mid \mathscr{F}_{t-1}\right) & =V\left(\mu_{t}\right) /(1+\varphi),
\end{aligned}
$$

respectively, where $V\left(\mu_{t}\right)=\mu_{t}\left(1-\mu_{t}\right)$ is the variance function and $\varphi$ can be interpreted as a precision parameter (reciprocal of dispersion).

Rocha and Cribari-Neto [30] introduced a dynamic model that can be used to model the behavior of variables that assume values in the standard unit interval. Their model, however, cannot be used when the variable of interest is subject to stochastic seasonal fluctuations. We shall now extend their model so that it can be used when such 
fluctuations do exist. Our interest lies in modeling the conditional mean of $y_{t}$.

The proposed beta seasonal autoregressive moving average model, $\beta \operatorname{SARMA}(p, q) \times(P, Q)_{S}$, is given by

$$
\Phi\left(B^{S}\right) \phi(B) g\left(y_{t}\right)=\beta+\Theta\left(B^{S}\right) \theta(B) r_{t},
$$

where $\beta \in \mathbb{R}$ is a constant, $r_{t}=g\left(y_{t}\right)-g\left(\mu_{t}\right)$ is the error term, $g(\cdot)$ is a strictly monotone and twice differentiable link function such that $g:(0,1) \rightarrow \mathbb{R}, \phi(B)=1-\phi_{1} B-\phi_{2} B^{2}-\cdots-\phi_{p} B^{p}$ is the autoregressive polynomial of order $p, \theta(B)=1-\theta_{1} B-\theta_{2} B^{2}-\cdots-\theta_{q} B^{q}$ is the moving average polynomial of order $q, \Phi\left(B^{S}\right)=1-\Phi_{1} B^{S}-\Phi_{2} B^{2 S}-$ $\cdots-\Phi_{P} B^{P S}$ is the seasonal autoregressive polynomial of order $P, \Theta\left(B^{S}\right)=1-\Theta_{1} B^{S}-\Theta_{2} B^{2 S}-\cdots-\Theta_{Q} B^{Q S}$ is the seasonal moving average polynomial of order $Q, B$ is the backshift operator such that $B^{d} g\left(y_{t}\right)=g\left(y_{t-d}\right)$, and $B^{d} r_{t}=r_{t-d}$ for a nonnegative integer $d$; and $S$ is the seasonality frequency (typically, $S=12$ for monthly data and $S=4$ for quarterly data).

\subsection{Some particular cases}

The class of $\beta \operatorname{SARMA}(p, q) \times(P, Q)_{S}$ contains several important models as particular cases. Some of them are listed below.

\subsection{1 $\beta \operatorname{ARMA}(1,1)$ or $\beta \operatorname{SARMA}(1,1) \times(0,0)_{S}$}

The $\beta \operatorname{ARMA}(1,1)$ can be written as

$$
\begin{aligned}
& \phi(B) g\left(y_{t}\right)=\beta+\theta(B) r_{t} \\
& \left(1-\phi_{1} B\right) g\left(y_{t}\right)=\beta+\left(1-\theta_{1} B\right) r_{t} \\
& g\left(y_{t}\right)-\phi_{1} g\left(y_{t-1}\right)=\beta+r_{t}-\theta_{1} r_{t-1} \\
& g\left(y_{t}\right)-\phi_{1} g\left(y_{t-1}\right)=\beta+\left[g\left(y_{t}\right)-g\left(\mu_{t}\right)\right]-\theta_{1} r_{t-1} \\
& g\left(\mu_{t}\right)=\beta+\phi_{1} g\left(y_{t-1}\right)-\theta_{1} r_{t-1}=\eta_{t},
\end{aligned}
$$

where $g\left(\mu_{t}\right)=\eta_{t}$ is the linear predictor.

\subsection{2 $\beta \operatorname{ARMA}(p, q)$}

Using backshift operator the $\beta \operatorname{ARMA}(p, q)$ model can be written as

$$
\begin{aligned}
& \phi(B) g\left(y_{t}\right)=\beta+\theta(B) r_{t} \\
& \left(1-\phi_{1} B-\cdots-\phi_{p} B^{p}\right) g\left(y_{t}\right)=\beta+\left(1-\theta_{1} B-\cdots-\theta_{q} B^{q}\right) r_{t}
\end{aligned}
$$




$$
\begin{aligned}
& g\left(y_{t}\right)-\phi_{1} g\left(y_{t-1}\right)-\cdots-\phi_{p} g\left(y_{t-p}\right)=\beta+r_{t}-\theta_{1} r_{t-1}-\cdots-\theta_{q} r_{t-q} \\
& g\left(y_{t}\right)-\phi_{1} g\left(y_{t-1}\right)-\cdots-\phi_{p} g\left(y_{t-p}\right)=\beta+\left[g\left(y_{t}\right)-g\left(\mu_{t}\right)\right]-\theta_{1} r_{t-1}-\cdots-\theta_{q} r_{t-q} \\
& g\left(\mu_{t}\right)=\beta+\sum_{i=1}^{p} \phi_{i} g\left(y_{t-i}\right)-\sum_{j=1}^{q} \theta_{j} r_{t-j} .
\end{aligned}
$$

Such a model is thus a special case of the more general class of models we propose in this paper.

\subsection{3 $\beta \operatorname{SARMA}(1,1) \times(1,1)_{12}$}

The $\beta \operatorname{SARMA}(1,1) \times(1,1)_{12}$ model can be written as

$$
\begin{aligned}
& \Phi\left(B^{12}\right) \phi(B) g\left(y_{t}\right)=\beta+\Theta\left(B^{12}\right) \theta(B) r_{t} \\
& \left(1-\Phi_{1} B^{12}\right)\left(1-\phi_{1} B\right) g\left(y_{t}\right)=\beta+\left(1-\Theta_{1} B^{12}\right)\left(1-\theta_{1} B\right) r_{t} \\
& g\left(y_{t}\right)-\phi_{1} g\left(y_{t-1}\right)-\Phi_{1} g\left(y_{t-12}\right)+\phi_{1} \Phi_{1} g\left(y_{t-13}\right)=\beta+r_{t}-\theta_{1} r_{t-1}-\Theta_{1} r_{t-12}+\theta_{1} \Theta_{1} r_{t-13} \\
& g\left(\mu_{t}\right)=\beta+\phi_{1} g\left(y_{t-1}\right)+\Phi_{1} g\left(y_{t-12}\right)-\phi_{1} \Phi_{1} g\left(y_{t-13}\right)-\theta_{1} r_{t-1}-\Theta_{1} r_{t-12}+\theta_{1} \Theta_{1} r_{t-13} .
\end{aligned}
$$

\subsection{4 $\beta \operatorname{SARMA}(2,0) \times(2,0)_{12}$}

The $\beta \operatorname{SARMA}(2,0) \times(2,0)_{12}$ model is given by

$$
\begin{aligned}
& \Phi\left(B^{12}\right) \phi(B) g\left(y_{t}\right)=\beta+r_{t} \\
& \left(1-\Phi_{1} B^{12}-\Phi_{2} B^{24}\right)\left(1-\phi_{1} B-\phi_{2} B^{2}\right) g\left(y_{t}\right)=\beta+r_{t} \\
& g\left(y_{t}\right)-\phi_{1} g\left(y_{t-1}\right)-\phi_{2} g\left(y_{t-2}\right)-\Phi_{1} g\left(y_{t-12}\right)-\Phi_{2} g\left(y_{t-24}\right)+\phi_{1} \Phi_{1} g\left(y_{t-13}\right) \\
& \quad+\phi_{2} \Phi_{1} g\left(y_{t-14}\right)+\phi_{1} \Phi_{2} g\left(y_{t-25}\right)+\phi_{2} \Phi_{2} g\left(y_{t-26}\right)=\beta+r_{t} \\
& g\left(\mu_{t}\right)=\beta+\phi_{1} g\left(y_{t-1}\right)+\phi_{2} g\left(y_{t-2}\right)+\Phi_{1} g\left(y_{t-12}\right)+\Phi_{2} g\left(y_{t-24}\right)-\phi_{1} \Phi_{1} g\left(y_{t-13}\right)-\phi_{2} \Phi_{1} g\left(y_{t-14}\right) \\
& \quad-\phi_{1} \Phi_{2} g\left(y_{t-25}\right)-\phi_{2} \Phi_{2} g\left(y_{t-26}\right) .
\end{aligned}
$$

\section{Parameter estimation}

Parameter estimation can be carried out by conditional maximum likelihood [50]. Let $\boldsymbol{\gamma}=\left(\boldsymbol{\beta}, \boldsymbol{\phi}^{\top}, \boldsymbol{\theta}^{\top}, \boldsymbol{\Phi}^{\top}, \boldsymbol{\Theta}^{\top}, \boldsymbol{\varphi}\right)^{\top}$ be the $k$-dimensional parameter vector, where $\boldsymbol{\phi}=\left(\phi_{1}, \ldots, \phi_{p}\right)^{\top}, \boldsymbol{\theta}=\left(\theta_{1}, \ldots, \theta_{q}\right)^{\top}, \boldsymbol{\Phi}=\left(\Phi_{1}, \ldots, \Phi_{P}\right)^{\top}, \boldsymbol{\Theta}=$ $\left(\Theta_{1}, \ldots, \Theta_{Q}\right)^{\top}$, and $k=p+q+P+Q+2$. The conditional maximum likelihood estimators (CMLE) of $\gamma$ are obtained by maximizing the logarithm of the conditional likelihood function. The log-likelihood function for the parameter 
vector $\gamma$ conditional on the $m$ initial observations, where $m=\max (P S+p, Q S+q)$, can be written as

$$
\ell=\ell(\boldsymbol{\gamma} ; \boldsymbol{y})=\sum_{t=m+1}^{n} \log f\left(y_{t} \mid \mathscr{F}_{t-1}\right)=\sum_{t=m+1}^{n} \ell_{t}\left(\mu_{t}, \varphi\right)
$$

where $\ell_{t}\left(\mu_{t}, \varphi\right)=\log \Gamma(\varphi)-\log \Gamma\left(\mu_{t} \varphi\right)-\log \Gamma\left(\left(1-\mu_{t}\right) \varphi\right)+\left(\mu_{t} \varphi-1\right) \log y_{t}+\left\{\left(1-\mu_{t}\right) \varphi-1\right\} \log \left(1-y_{t}\right)$.

\subsection{Conditional score vector}

Let $\boldsymbol{\lambda}=\left(\boldsymbol{\beta}, \boldsymbol{\phi}^{\top}, \boldsymbol{\theta}^{\top}, \boldsymbol{\Phi}^{\top}, \boldsymbol{\Theta}^{\top}\right)^{\top}$. Differentiation of the conditional log-likelihood function given in (2) with respect to $i$ th element of $\lambda, \lambda_{i}$, with $i=1, \ldots,(k-1)$, yields

$$
\frac{\partial \ell}{\partial \lambda_{i}}=\sum_{t=m+1}^{n} \frac{\partial \ell_{t}\left(\mu_{t}, \varphi\right)}{\partial \mu_{t}} \frac{d \mu_{t}}{d \eta_{t}} \frac{\partial \eta_{t}}{\partial \lambda_{i}}
$$

Since

$$
\frac{\partial \ell_{t}\left(\mu_{t}, \varphi\right)}{\partial \mu_{t}}=\varphi\left\{\log \frac{y_{t}}{1-y_{t}}-\left[\psi\left(\mu_{t} \varphi\right)-\psi\left(\left(1-\mu_{t}\right) \varphi\right)\right]\right\}
$$

where $\psi(\cdot)$ is the digamma function, it follows that

$$
\frac{\partial \ell}{\partial \lambda_{i}}=\varphi \sum_{t=m+1}^{n}\left(y_{t}^{*}-\mu_{t}^{*}\right) \frac{1}{g^{\prime}\left(\mu_{t}\right)} \frac{\partial \eta_{t}}{\partial \lambda_{i}}
$$

where $y_{t}^{*}=\log \left\{y_{t} /\left(1-y_{t}\right)\right\}, \mu_{t}^{*}=\psi\left(\mu_{t} \varphi\right)-\psi\left(\left(1-\mu_{t}\right) \varphi\right)$.

When $\lambda_{i}=\beta$, the linear predictor derivative is

$$
\frac{\partial \eta_{t}}{\partial \beta}=1-\sum_{j=1}^{q} \theta_{j} \frac{\partial r_{t-j}}{\partial \beta}-\sum_{J=1}^{Q} \Theta_{J} \frac{\partial r_{t-J S}}{\partial \beta}+\sum_{j=1}^{q} \sum_{J=1}^{Q} \theta_{j} \Theta_{J} \frac{\partial r_{t-(j+J S)}}{\partial \beta}
$$

Since that $r_{t}=g\left(y_{t}\right)-\eta_{t}$, we obtain

$$
\frac{\partial \eta_{t}}{\partial \beta}=1+\sum_{j=1}^{q} \theta_{j} \frac{\partial \eta_{t-j}}{\partial \beta}+\sum_{J=1}^{Q} \Theta_{J} \frac{\partial \eta_{t-J S}}{\partial \beta}-\sum_{j=1}^{q} \sum_{J=1}^{Q} \theta_{j} \Theta_{J} \frac{\partial \eta_{t-(j+J S)}}{\partial \beta}
$$

The linear predictor derivatives with respect to the remaining parameters are given by

$$
\begin{gathered}
\frac{\partial \eta_{t}}{\partial \phi_{i}}=g\left(y_{t-i}\right) \Phi\left(B^{S}\right)+\sum_{j=1}^{q} \theta_{j} \frac{\partial \eta_{t-j}}{\partial \phi_{i}}+\sum_{J=1}^{Q} \Theta_{J} \frac{\partial \eta_{t-J S}}{\partial \phi_{i}}-\sum_{j=1}^{q} \sum_{J=1}^{Q} \theta_{j} \Theta_{J} \frac{\partial \eta_{t-(j+J S)}}{\partial \phi_{i}} \\
\frac{\partial \eta_{t}}{\partial \Phi_{I}}=g\left(y_{t-I S}\right) \phi(B)+\sum_{j=1}^{q} \theta_{j} \frac{\partial \eta_{t-j}}{\partial \Phi_{I}}+\sum_{J=1}^{Q} \Theta_{J} \frac{\partial \eta_{t-J S}}{\partial \Phi_{I}}-\sum_{j=1}^{q} \sum_{J=1}^{Q} \theta_{j} \Theta_{J} \frac{\partial \eta_{t-(j+J S)}}{\partial \Phi_{I}} \\
\frac{\partial \eta_{t}}{\partial \theta_{j}}=-r_{t-j} \Theta\left(B^{S}\right)+\sum_{i=1}^{q} \theta_{i} \frac{\partial \eta_{t-i}}{\partial \theta_{j}}+\sum_{J=1}^{Q} \Theta_{J} \frac{\partial \eta_{t-J S}}{\partial \theta_{j}}-\sum_{i=1}^{q} \sum_{J=1}^{Q} \theta_{i} \Theta_{J} \frac{\partial \eta_{t-(i+J S)}}{\partial \theta_{j}}
\end{gathered}
$$




$$
\frac{\partial \eta_{t}}{\partial \Theta_{J}}=-r_{t-J S} \theta(B)+\sum_{j=1}^{q} \theta_{j} \frac{\partial \eta_{t-j}}{\partial \Theta_{J}}+\sum_{i=1}^{Q} \Theta_{i} \frac{\partial \eta_{t-i S}}{\partial \Theta_{J}}-\sum_{j=1}^{q} \sum_{i=1}^{Q} \theta_{j} \Theta_{i} \frac{\partial \eta_{t-(j+i S)}}{\partial \Theta_{J}}
$$

As in [51] and [52], when there are no moving average components (i.e., when $\theta_{j}=0$ and $\Theta_{J}=0$ for all $j$ and $J$ ) no recursion is necessary to evaluate $\eta_{t}$ and its partial derivatives. When the model includes moving average dynamics (ordinary or seasonal), we suggest using $\eta_{t}=g\left(y_{t}\right)$ and setting all linear predictor derivatives equal to zero for the initial cases, as in [52].

Finally, differentiation of $\ell(\boldsymbol{\gamma} ; \boldsymbol{y})$ with respect to the precision parameter $\varphi$ yields

$$
\frac{\partial \ell}{\partial \varphi}=\sum_{t=m+1}^{n}\left\{\mu_{t}\left(y_{t}^{*}-\mu_{t}^{*}\right)+\log \left(1-y_{t}\right)-\psi\left(\left(1-\mu_{t}\right) \varphi\right)+\psi(\varphi)\right\}
$$

Therefore, the elements of the score vector $\boldsymbol{U}(\boldsymbol{\gamma})=\left(U_{\beta}(\boldsymbol{\gamma}), \boldsymbol{U}_{\phi}(\boldsymbol{\gamma})^{\top}, \boldsymbol{U}_{\theta}(\boldsymbol{\gamma})^{\top}, \boldsymbol{U}_{\Phi}(\boldsymbol{\gamma})^{\top}, \boldsymbol{U}_{\Theta}(\boldsymbol{\gamma})^{\top}, U_{\varphi}(\boldsymbol{\gamma})\right)^{\top}$ can be written, in matrix form, as

$$
\begin{aligned}
& U_{\beta}(\boldsymbol{\gamma})=\varphi \boldsymbol{a}^{\top} \boldsymbol{T}\left(\boldsymbol{y}^{*}-\boldsymbol{\mu}^{*}\right), \\
& \boldsymbol{U}_{\phi}(\boldsymbol{\gamma})=\varphi \boldsymbol{A}^{\top} \boldsymbol{T}\left(\boldsymbol{y}^{*}-\boldsymbol{\mu}^{*}\right), \\
& \boldsymbol{U}_{\boldsymbol{\Phi}}(\boldsymbol{\gamma})=\varphi \mathcal{A}^{\top} \boldsymbol{T}\left(\boldsymbol{y}^{*}-\boldsymbol{\mu}^{*}\right), \\
& \boldsymbol{U}_{\boldsymbol{\theta}}(\boldsymbol{\gamma})=\varphi \boldsymbol{M}^{\top} \boldsymbol{T}\left(\boldsymbol{y}^{*}-\boldsymbol{\mu}^{*}\right), \\
& \boldsymbol{U}_{\Theta}(\boldsymbol{\gamma})=\varphi \mathcal{M}^{\top} \boldsymbol{T}\left(\boldsymbol{y}^{*}-\boldsymbol{\mu}^{*}\right), \\
& U_{\varphi}(\boldsymbol{\gamma})=\sum_{t=m+1}^{n}\left\{\mu_{t}\left(y_{t}^{*}-\mu_{t}^{*}\right)+\log \left(1-y_{t}\right)-\psi\left(\left(1-\mu_{t}\right) \varphi\right)+\psi(\varphi)\right\},
\end{aligned}
$$

where $\boldsymbol{y}^{*}=\left(y_{m+1}^{*}, \ldots, y_{n}^{*}\right)^{\top}, \boldsymbol{\mu}^{*}=\left(\mu_{m+1}^{*}, \ldots, \mu_{n}^{*}\right)^{\top}, \boldsymbol{T}=\operatorname{diag}\left\{1 / g^{\prime}\left(\mu_{m+1}\right), \ldots, 1 / g^{\prime}\left(\mu_{n}\right)\right\}, \boldsymbol{a}=\left(\frac{\partial \eta_{m+1}}{\partial \beta}, \ldots, \frac{\partial \eta_{n}}{\partial \beta}\right)^{\top}$, $\boldsymbol{A}$ is an $(n-m) \times p$ matrix whose $(i, j)$ element is given by $\partial \eta_{i+m} / \partial \phi_{j}, \mathcal{A}$ is an $(n-m) \times P$ matrix whose $(i, j)$ element equals $\partial \eta_{i+m} / \partial \Phi_{j}, \boldsymbol{M}$ is an $(n-m) \times q$ matrix whose $(i, j)$ element is given by $\partial \eta_{i+m} / \partial \theta_{j}$, and $\mathcal{M}$ is an $(n-m) \times Q$ matrix whose $(i, j)$ element is $\partial \eta_{i+m} / \partial \Theta_{j}$,

The conditional maximum likelihood estimator of $\boldsymbol{\gamma}$ is obtained as the solution to the following system of equations:

$$
\boldsymbol{U}(\boldsymbol{\gamma})=\mathbf{0},
$$

where $\mathbf{0}$ is the $k \times 1$ vector of zeros. The solution to the above system of nonlinear equation cannot be written in closed form. Conditional maximum likelihood estimates can be obtained by numerically maximizing the conditional log-likelihood function using a Newton or quasi-Newton nonlinear optimization algorithm; see, e.g., [53]. In what follows, we shall use the quasi-Newton algorithm known as Broyden-Fletcher-Goldfarb-Shanno (BFGS); for details, see [54]. 


\subsection{Conditional information matrix}

The CMLE asymptotic covariance matrix, which can be used to construct confidence intervals, is given by the inverse of the conditional Fisher information matrix. In order to obtain such a matrix we need to compute the expected values of all second order derivatives.

Let $\boldsymbol{\lambda}=\left(\beta, \boldsymbol{\phi}^{\top}, \boldsymbol{\theta}^{\top}, \boldsymbol{\Phi}^{\top}, \boldsymbol{\Theta}^{\top}\right)^{\top}$. It can be shown that

$$
\begin{aligned}
\frac{\partial^{2} \ell}{\partial \lambda_{i} \partial \lambda_{j}} & =\sum_{t=m+1}^{n} \frac{\partial}{\partial \mu_{t}}\left(\frac{\partial \ell_{t}\left(\mu_{t}, \varphi\right)}{\partial \mu_{t}} \frac{d \mu_{t}}{d \eta_{t}} \frac{\partial \eta_{t}}{\partial \lambda_{j}}\right) \frac{d \mu_{t}}{d \eta_{t}} \frac{\partial \eta_{t}}{\partial \lambda_{i}} \\
& =\sum_{t=m+1}^{n}\left[\frac{\partial^{2} \ell_{t}\left(\mu_{t}, \varphi\right)}{\partial \mu_{t}^{2}} \frac{d \mu_{t}}{d \eta_{t}} \frac{\partial \eta_{t}}{\partial \lambda_{j}}+\frac{\partial \ell_{t}\left(\mu_{t}, \varphi\right)}{\partial \mu_{t}} \frac{\partial}{\partial \mu_{t}}\left(\frac{d \mu_{t}}{d \eta_{t}} \frac{\partial \eta_{t}}{\partial \lambda_{j}}\right)\right] \frac{d \mu_{t}}{d \eta_{t}} \frac{\partial \eta_{t}}{\partial \lambda_{i}}
\end{aligned}
$$

for $i=1, \ldots,(k-1)$ and $j=1, \ldots,(k-1)$, where $k=p+q+P+Q+2$.

Under the usual regularity conditions, it follows that $\mathbb{E}\left(\partial \ell_{t}\left(\mu_{t}, \varphi\right) / \partial \mu_{t} \mid \mathscr{F}_{t-1}\right)=0$. Thus,

$$
\mathbb{E}\left(\frac{\partial^{2} \ell}{\partial \lambda_{i} \partial \lambda_{j}} \mid \mathscr{F}_{t-1}\right)=\sum_{t=m+1}^{n} \mathbb{E}\left(\frac{\partial^{2} \ell_{t}\left(\mu_{t}, \varphi\right)}{\partial \mu_{t}^{2}} \mid \mathscr{F}_{t-1}\right)\left(\frac{d \mu_{t}}{d \eta_{t}}\right)^{2} \frac{\partial \eta_{t}}{\partial \lambda_{j}} \frac{\partial \eta_{t}}{\partial \lambda_{i}}
$$

By differentiating (3) twice with respect to $\mu_{t}$, we obtain

$$
\frac{\partial^{2} \ell_{t}\left(\mu_{t}, \varphi\right)}{\partial \mu_{t}^{2}}=-\varphi^{2}\left\{\psi^{\prime}\left(\mu_{t} \varphi\right)+\psi^{\prime}\left[\left(1-\mu_{t}\right) \varphi\right]\right\}
$$

Furthermore,

$$
\mathbb{E}\left(\frac{\partial^{2} \ell}{\partial \lambda_{i} \partial \lambda_{j}} \mid \mathscr{F}_{t-1}\right)=-\sum_{t=m+1}^{n} \frac{w_{t}}{g^{\prime}\left(\mu_{t}\right)^{2}} \frac{\partial \eta_{t}}{\partial \lambda_{j}} \frac{\partial \eta_{t}}{\partial \lambda_{i}}
$$

where $w_{t}=\varphi^{2}\left\{\psi^{\prime}\left(\mu_{t} \varphi\right)+\psi^{\prime}\left[\left(1-\mu_{t}\right) \varphi\right]\right\}$. Notice that all first derivatives $\partial \eta_{t} / \partial \lambda_{i}$ have already been presented in Section 3.1

Differentiation of (4) with respect to $\lambda_{i}, i=1, \ldots,(k-1)$, yields

$$
\frac{\partial^{2} \ell}{\partial \varphi \partial \lambda_{i}}=\sum_{t=m+1}^{n}\left[\left(y_{t}^{*}-\mu_{t}^{*}\right)-\varphi \frac{\partial \mu_{t}^{*}}{\partial \varphi}\right] \frac{1}{g^{\prime}\left(\mu_{t}\right)} \frac{\partial \eta_{t}}{\partial \lambda_{i}}
$$

where $\partial \mu_{t}^{*} / \partial \varphi=\psi^{\prime}\left(\mu_{t} \varphi\right) \mu_{t}-\psi^{\prime}\left[\left(1-\mu_{t}\right) \varphi\right]\left(1-\mu_{t}\right)$. Under the usual regularity conditions, we have $\mathbb{E}\left(y_{t}^{*} \mid \mathscr{F}_{t-1}\right)=$ $\mu_{t}^{*}$, and thus

$$
\mathbb{E}\left(\frac{\partial^{2} \ell}{\partial \varphi \partial \lambda_{i}} \mid \mathscr{F}_{t-1}\right)=-\sum_{t=m+1}^{n} \frac{c_{t}}{g^{\prime}\left(\mu_{t}\right)} \frac{\partial \eta_{t}}{\partial \lambda_{i}}
$$

where $c_{t}=\varphi \frac{\partial \mu_{t}^{*}}{\partial \varphi}=\varphi\left\{\psi^{\prime}\left(\mu_{t} \varphi\right) \mu_{t}-\psi^{\prime}\left[\left(1-\mu_{t}\right) \varphi\right]\left(1-\mu_{t}\right)\right\}$. 
Finally, the expected value of the second order derivative of $\ell(\gamma ; y)$ with respect to $\varphi$ is given by

$$
\mathbb{E}\left(\frac{\partial^{2} \ell}{\partial \varphi^{2}} \mid \mathscr{F}_{t-1}\right)=-\sum_{t=m+1}^{n} d_{t},
$$

where $d_{t}=\psi^{\prime}\left(\mu_{t} \phi\right) \mu_{t}^{2}+\psi^{\prime}\left(\left(1-\mu_{t}\right) \phi\right)\left(1-\mu_{t}\right)^{2}-\psi^{\prime}(\phi)$.

Let $\boldsymbol{W}=\operatorname{diag}\left\{w_{m+1}, \ldots, w_{n}\right\}, \boldsymbol{C}=\operatorname{diag}\left\{c_{m+1}, \ldots, c_{n}\right\}$, and $\boldsymbol{D}=\operatorname{diag}\left\{d_{m+1}, \ldots, d_{n}\right\}$. The joint conditional Fisher information matrix for $\boldsymbol{\gamma}$ is

$$
\boldsymbol{K}=\boldsymbol{K}(\boldsymbol{\gamma})=\left(\begin{array}{cccccc}
\boldsymbol{K}_{(\beta, \beta)} & \boldsymbol{K}_{(\beta, \phi)} & \boldsymbol{K}_{(\beta, \Phi)} & \boldsymbol{K}_{(\beta, \theta)} & \boldsymbol{K}_{(\beta, \Theta)} & \boldsymbol{K}_{(\beta, \varphi)} \\
\boldsymbol{K}_{(\phi, \beta)} & \boldsymbol{K}_{(\phi, \phi)} & \boldsymbol{K}_{(\phi, \Phi)} & \boldsymbol{K}_{(\phi, \theta)} & \boldsymbol{K}_{(\phi, \Theta)} & \boldsymbol{K}_{(\phi, \varphi)} \\
\boldsymbol{K}_{(\Phi, \beta)} & \boldsymbol{K}_{(\Phi, \phi)} & \boldsymbol{K}_{(\Phi, \Phi)} & \boldsymbol{K}_{(\Phi, \theta)} & \boldsymbol{K}_{(\Phi, \Theta)} & \boldsymbol{K}_{(\Phi, \varphi)} \\
\boldsymbol{K}_{(\theta, \beta)} & \boldsymbol{K}_{(\theta, \phi)} & \boldsymbol{K}_{(\theta, \Phi)} & \boldsymbol{K}_{(\theta, \theta)} & \boldsymbol{K}_{(\theta, \Theta)} & \boldsymbol{K}_{(\theta, \varphi)} \\
\boldsymbol{K}_{(\Theta, \beta)} & \boldsymbol{K}_{(\Theta, \phi)} & \boldsymbol{K}_{(\Theta, \Phi)} & \boldsymbol{K}_{(\Theta, \theta)} & \boldsymbol{K}_{(\Theta, \Theta)} & \boldsymbol{K}_{(\Theta, \varphi)} \\
\boldsymbol{K}_{(\varphi, \beta)} & \boldsymbol{K}_{(\varphi, \phi)} & \boldsymbol{K}_{(\varphi, \Phi)} & \boldsymbol{K}_{(\varphi, \theta)} & \boldsymbol{K}_{(\varphi, \Theta)} & K_{(\varphi, \varphi)}
\end{array}\right),
$$

where $K_{(\beta, \beta)}=\boldsymbol{a}^{\top} \boldsymbol{W} \boldsymbol{T}^{2} \boldsymbol{a}, \boldsymbol{K}_{(\beta, \phi)}=\boldsymbol{K}_{(\phi, \beta)}^{\top}=\boldsymbol{a}^{\top} \boldsymbol{W} \boldsymbol{T}^{2} \boldsymbol{A}, \boldsymbol{K}_{(\beta, \Phi)}=\boldsymbol{K}_{(\Phi, \beta)}^{\top}=\boldsymbol{a}^{\top} \boldsymbol{W} \boldsymbol{T}^{2} \mathcal{A}, \boldsymbol{K}_{(\beta, \theta)}=\boldsymbol{K}_{(\theta, \beta)}^{\top}=$ $\boldsymbol{a}^{\top} \boldsymbol{W} \boldsymbol{T}^{2} \boldsymbol{M}, \boldsymbol{K}_{(\beta, \Theta)}=\boldsymbol{K}_{(\Theta, \beta)}^{\top}=\boldsymbol{a}^{\top} \boldsymbol{W} \boldsymbol{T}^{2} \mathcal{M}, \boldsymbol{K}_{(\beta, \varphi)}=\boldsymbol{K}_{(\varphi, \beta)}=\boldsymbol{a}^{\top} \boldsymbol{C T} \mathbf{1}, \boldsymbol{K}_{(\phi, \phi)}=\boldsymbol{A}^{\top} \boldsymbol{W} \boldsymbol{T}^{2} \boldsymbol{A}, \boldsymbol{K}_{(\phi, \Phi)}=\boldsymbol{K}_{(\Phi, \phi)}^{\top}=$ $\boldsymbol{A}^{\top} \boldsymbol{W} \boldsymbol{T}^{2} \mathcal{A}, \boldsymbol{K}_{(\phi, \theta)}=\boldsymbol{K}_{(\theta, \phi)}^{\top}=\boldsymbol{A}^{\top} \boldsymbol{W} \boldsymbol{T}^{2} \boldsymbol{M}, \boldsymbol{K}_{(\phi, \Theta)}=\boldsymbol{K}_{(\Theta, \phi)}^{\top}=\boldsymbol{A}^{\top} \boldsymbol{W} \boldsymbol{T}^{2} \mathcal{M}, \boldsymbol{K}_{(\phi, \varphi)}=\boldsymbol{K}_{(\varphi, \phi)}^{\top}=\boldsymbol{A}^{\top} \boldsymbol{C T} \mathbf{1}, \boldsymbol{K}_{(\Phi, \Phi)}=$ $\mathcal{A}^{\top} \boldsymbol{W} \boldsymbol{T}^{2} \mathcal{A}, \boldsymbol{K}_{(\Phi, \theta)}=\boldsymbol{K}_{(\theta, \Phi)}^{\top}=\mathcal{A}^{\top} \boldsymbol{W} \boldsymbol{T}^{2} \boldsymbol{M}, \boldsymbol{K}_{(\Phi, \Theta)}=\boldsymbol{K}_{(\Theta, \Phi)}^{\top}=\mathcal{A}^{\top} \boldsymbol{W} \boldsymbol{T}^{2} \mathcal{M}, \boldsymbol{K}_{(\Phi, \varphi)}=\boldsymbol{K}_{(\varphi, \Phi)}^{\top}=\mathcal{A}^{\top} \boldsymbol{C T} \mathbf{1}$, $\boldsymbol{K}_{(\theta, \theta)}=\boldsymbol{M}^{\top} \boldsymbol{W} \boldsymbol{T}^{2} \boldsymbol{M}, \boldsymbol{K}_{(\theta, \Theta)}=\boldsymbol{K}_{(\Theta, \theta)}^{\top}=\boldsymbol{M}^{\top} \boldsymbol{W} \boldsymbol{T}^{2} \mathcal{M}, \boldsymbol{K}_{(\theta, \varphi)}=\boldsymbol{K}_{(\varphi, \theta)}^{\top}=\boldsymbol{M}^{\top} \boldsymbol{C T} \mathbf{1}, \boldsymbol{K}_{(\Theta, \Theta)}=\mathcal{M}^{\top} \boldsymbol{W} \boldsymbol{T}^{2} \mathcal{M}$, $\boldsymbol{K}_{(\Theta, \varphi)}=\boldsymbol{K}_{(\varphi, \Theta)}^{\top}=\mathcal{M}^{\top} \boldsymbol{C T} \mathbf{1}, K_{(\varphi, \varphi)}=\operatorname{tr}(\boldsymbol{D})$, and $\mathbf{1}$ is the $(n-m) \times 1$ vector of ones. We note that the conditional Fisher information matrix is not block diagonal, and hence the parameters are not orthogonal [55].

Under some mild regularity conditions the conditional maximum likelihood estimates are consistent and asymptotically normally distributed [50]. Thus, in large sample sizes,

$$
\left.\left(\begin{array}{c}
\widehat{\beta} \\
\widehat{\boldsymbol{\phi}} \\
\widehat{\boldsymbol{\Phi}} \\
\widehat{\boldsymbol{\theta}} \\
\widehat{\boldsymbol{\Theta}} \\
\widehat{\varphi}
\end{array}\right) \sim \mathscr{N}_{k}\left(\begin{array}{c}
\beta \\
\boldsymbol{\phi} \\
\boldsymbol{\Phi} \\
\boldsymbol{\Theta} \\
\varphi
\end{array}\right), \boldsymbol{K}^{-1}\right)
$$

approximately, where $\widehat{\beta}, \widehat{\boldsymbol{\phi}}, \widehat{\boldsymbol{\Phi}}, \widehat{\boldsymbol{\theta}}, \widehat{\boldsymbol{\Theta}}$ and $\widehat{\varphi}$ are the CMLEs of $\beta, \boldsymbol{\phi}, \boldsymbol{\Phi}, \boldsymbol{\theta}, \boldsymbol{\Theta}$ and $\boldsymbol{\varphi}$, respectively. Notice that $\boldsymbol{K}^{-1}$ is the asymptotic covariance matrix of $\widehat{\gamma}$. 


\subsection{Confidence intervals and hypothesis testing inference}

Let $\gamma_{r}$ denote the $r$ th component of $\boldsymbol{\gamma}$. From $[5$, we have that

$$
\left(\widehat{\gamma}_{r}-\gamma_{r}\right)\left\{K(\widehat{\gamma})^{r r}\right\}^{-1 / 2} \sim \mathscr{N}(0,1)
$$

approximately, where $K(\widehat{\gamma})^{r r}$ is the $r$ th diagonal element of $\boldsymbol{K}^{-1}$. Let $z_{\delta}$ represent the $\delta$ standard normal quantile. A $100(1-\alpha) \%, 0<\alpha<1 / 2$, confidence interval for $\gamma_{r}, r=1, \ldots, k$, is

$$
\left[\widehat{\gamma}_{r}-z_{1-\alpha / 2}\left(K(\widehat{\gamma})^{r r}\right)^{1 / 2} ; \widehat{\gamma}_{r}+z_{1-\alpha / 2}\left(K(\widehat{\gamma})^{r r}\right)^{1 / 2}\right]
$$

Details on asymptotic confidence intervals can be found in [56] and [57].

The test for $\mathscr{H}_{0}: \gamma_{r}=\gamma_{r}^{0}$ against $\mathscr{H}_{1}: \gamma_{r} \neq \gamma_{r}^{0}$ can be based on the signed square root of Wald's statistic, which is given by [56]

$$
z=\frac{\widehat{\gamma}_{r}-\gamma_{r}^{0}}{\widehat{\operatorname{se}}\left(\widehat{\gamma}_{r}\right)}
$$

where the asymptotic standard error of the $\widehat{\gamma}_{r}$ is $\widehat{\operatorname{se}}\left(\widehat{\gamma}_{r}\right)=\left(K(\widehat{\gamma})^{r r}\right)^{1 / 2}$. Under $\mathscr{H}_{0}$, the limiting distribution of $z$ is standard normal.

It is possible to perform hypothesis testing inference using the the likelihood ratio [58], Rao's score [59], Wald [60], and gradient [61] tests. In large samples and under the null hypothesis, such test statistics are chi-squared distributed.

The Wald test for the presence of seasonal movements can be carried out as follows. The null and alternative hypotheses are

$$
\begin{array}{ll}
\mathscr{H}_{0}:\left(\Phi_{1}, \ldots, \Phi_{P}, \Theta_{1}, \ldots, \Theta_{Q}\right)^{\top}=\mathbf{0}_{P+Q} \quad \text { (non-seasonal), } \\
\mathscr{H}_{1}:\left(\Phi_{1}, \ldots, \Phi_{P}, \Theta_{1}, \ldots, \Theta_{Q}\right)^{\top} \neq \mathbf{0}_{P+Q} \quad \text { (seasonal), }
\end{array}
$$

where $\mathbf{0}_{P+Q}$ is the $(P+Q)$-vector of zeros. Under $\mathscr{H}_{0}$, there is no seasonal dynamics. Rejection of the null hypothesis indicates that seasonality must be accounted for. The Wald test statistics is

$$
W=\left(\widehat{\Phi}_{1}, \ldots, \widehat{\Phi}_{P}, \widehat{\Theta}_{1}, \ldots, \widehat{\Theta}_{Q}\right)\left(\boldsymbol{K}(\widehat{\boldsymbol{\gamma}})^{\Phi \Theta}\right)^{-1}\left(\widehat{\Phi}_{1}, \ldots, \widehat{\Phi}_{P}, \widehat{\Theta}_{1}, \ldots, \widehat{\Theta}_{Q}\right)^{\top}
$$

where $\boldsymbol{K}(\widehat{\gamma})^{\Phi \Theta}$ is the $(P+Q) \times(P+Q)$ block of the inverse of Fisher's information matrix relative to the seasonal parameters evaluated at $\hat{\gamma}$. Under standard regularity conditions and under the null hypothesis, $W$ is asymptotically chi-squared distribution with $P+Q$ degrees of freedom $\left(\chi_{P+Q}^{2}\right)$. Notice that in order to compute $W$ one only needs to 
estimate the non-null (seasonal) model.

\section{Model selection, diagnostic analysis and forecasting}

In what follows we present some model selection criteria that can be used for model identification and present some diagnostic tools for fitted $\beta$ SARMA models. Diagnostic checks can be applied to a fitted model to determine whether it fully captures the data dynamics. A fitted model that passes all diagnostic checks can then be used for out-of-sample forecasting.

\subsection{Model selection criteria}

Model selection can be based on Akaike's Information Criterion (AIC) [62]. Since the conditional log-likelihood is additive, using the idea introduced by [63] for bootstrapped likelihood cross validation, we propose the following modified AIC:

$$
\mathrm{MAIC}=-2 \hat{\ell}_{*}+2 k
$$

where $\hat{\ell}_{*}=\hat{\ell} \times n /(n-m)$ and $k=p+q+P+Q+2$ is the number of parameters in the model. When comparing models of different dimensions for different values of $m, \hat{\ell}_{*}$ can be interpreted as the sum of $n$ terms. Therefore, the MAIC does not incorrectly penalize models with larger values of $m$.

The MAIC aims at estimating the expected conditional log-likelihood using a bias correction $(2 k)$ for the maximized conditional log-likelihood function. When $2 k$ in 6 is replaced by $\log (n) k$ we obtain the modified Schwarz Information Criterion (MSIC) [64]; when it is replaced by $\log [\log (n)] k$, the modified Hannan and Quinn Information Criterion (MHQ) [65] is obtained. Alternative choices can be considered for the bias correcting term, such as in [66, 67, 68] or the bootstrapped versions in 69, 70, 71, 72, 73, 74, 75].

\subsection{Deviance}

The deviance is defined as twice the difference between the conditional log-likelihood evaluated at the saturated model (for which $\tilde{\mu}_{t}=y_{t}$ ) and at the fitted model. That is, the deviance is given by

$$
D=2[\tilde{\ell}-\hat{\ell}]
$$

where $\hat{\ell}=\sum_{t=m+1}^{n} \ell_{t}\left(\hat{\mu}_{t}, \hat{\varphi}\right)$ and $\tilde{\ell}=\sum_{t=m+1}^{n} \ell_{t}\left(y_{t}, \hat{\varphi}\right)$. When the fitted model is correctly specified, $D$ is approximately distributed as $\chi_{n-m-k}^{2}[14,21]$. It is customary to divide the deviance by $n-m-k$. There is evidence of incorrect model specification when $D /(n-m-k)$ is considerably larger than one [76]. 


\subsection{Residuals}

Residual analysis is important for determining whether the model at hand provides a good fit [21]. Visual inspection of a time series residuals plot is an indispensable first step when assessing goodness-of-fit [2]. Various types of residuals are currently available [77]. Since the model we propose is an extension of the beta regression model [24] for time series analysis, the residual used in beta regression diagnostics can also be used here. For details on residuals and diagnostics tools in beta regression models, see [78, 79]. For details on time series model residuals, see [21].

At the outset, we define the following standardized residual:

$$
\hat{r}_{t}^{1}=\frac{y_{t}-\widehat{\mu}_{t}}{\sqrt{\widehat{\operatorname{Var}}\left(y_{t}\right)}}=\frac{y_{t}-\widehat{\mu}_{t}}{\sqrt{V\left(\widehat{\mu}_{t}\right) /(1+\widehat{\varphi})}} .
$$

Considering the predictor scale, we define the following standardized residual 2:

$$
\hat{r}_{t}^{2}=\frac{g\left(y_{t}\right)-\widehat{\eta}_{t}}{\sqrt{\left(g^{\prime}\left(\widehat{\mu}_{t}\right)\right)^{2} V\left(\widehat{\mu}_{t}\right) /(1+\widehat{\varphi})}} .
$$

Using a Taylor series expansion, in [30] is shown that $\operatorname{Var}\left(g\left(y_{t}\right)\right) \approx\left(g^{\prime}\left(\mu_{t}\right)\right)^{2} V\left(\mu_{t}\right) /(1+\varphi)$.

A more sophisticated residual is the standardized weighted residual introduced by [79], which is given by

$$
\hat{r}_{t}^{w}=\frac{y_{t}^{*}-\widehat{\mu}_{t}^{*}}{\sqrt{\widehat{\operatorname{Var}}\left(y_{t}^{*}\right)}}=\frac{y_{t}^{*}-\widehat{\mu}_{t}^{*}}{\sqrt{\psi^{\prime}\left(\widehat{\mu}_{t} \widehat{\varphi}\right)+\psi^{\prime}\left[\left(1-\widehat{\mu}_{t}\right) \hat{\varphi}\right]}} .
$$

The authors have shown that $\operatorname{Var}\left(y_{t}^{*}\right)=\psi^{\prime}\left(\mu_{t} \varphi\right)+\psi^{\prime}\left[\left(1-\mu_{t}\right) \varphi\right]$. Under correct model specification, the distribution of such a residual is approximately standard normal.

\subsection{White noise tests}

When the model is correctly specified the residuals are expected to behave as white noise, i.e., they are expected to be serially uncorrelated and follow a zero mean and constant variance process [21]. Let $\hat{r}_{m+1}^{w}, \ldots, \hat{r}_{n}^{w}$ be the standardized weighted residuals obtained from the fitted model. The residual autocorrelation function (ACF) is

$$
\widehat{\rho}(i)=\frac{\sum_{t=m+1}^{n-i}\left(\hat{r}_{t}^{w}-\bar{r}^{w}\right)\left(\hat{r}_{t+i}^{w}-\bar{r}^{w}\right)}{\sum_{t=m+1}^{n-i}\left(\hat{r}_{t}^{w}-\bar{r}^{w}\right)^{2}}, \quad i=0,1, \ldots,
$$

where $\bar{r}^{w}=(n-m)^{-1} \sum_{t=m+1}^{n} \hat{r}_{t}^{w}$. When $i>1$ and $n$ is sufficiently large, the distribution of $\widehat{\rho}(i)$ is approximately normal with zero mean and variance $1 /(n-m)$ [21 80, 2]. Hence, plots the residuals ACF with horizontal lines at $\pm 1.96 / \sqrt{(n-m)}$ can be used for assessing whether the residuals display white noise behavior [21]. It is expected that $95 \%$ of residuals autocorrelations lie inside the interval $[-1.96 / \sqrt{(n-m)}, 1.96 / \sqrt{(n-m)}]$.

It is also possible to test the null hypothesis that the first $b$ residual autocorrelations are equal to zero. To that end, 
the following test statistic can be used [81]:

$$
Q_{1}=(n-m)(n-m+2) \sum_{i=1}^{b} \frac{[\widehat{\rho}(i)]^{2}}{n-m-i}
$$

Alternatively, it is possible to base the test statistic on residual partial autocorrelations [82]:

$$
Q_{2}=(n-m)(n-m+2) \sum_{i=1}^{b} \frac{[p(i)]^{2}}{n-m-i}
$$

where $p(i)$ is the $i$ th partial residual autocorrelation.

The critical value used in either test is obtained from the test statistic asymptotic null distribution, namely $\chi_{b-p-q-P-Q}^{2}$. The null hypothesis is rejected at nominal level $\alpha$ if $Q_{j}>\chi_{1-\alpha, b-p-q-P-Q}^{2}, j=1,2$, where $Q_{j}>\chi_{1-\alpha, b-p-q-P-Q}^{2}$ is the $1-\alpha \chi_{b-p-q-P-Q}^{2}$ quantile. Based on pilot simulations and on a rule-of-thumb available in the literature [83], we suggest using $b=\max (10,2 S)$, where $S$ is the seasonality frequency.

\subsection{Forecasting}

Estimates of $\mu_{t}, \widehat{\mu}_{t}$, for $t=m, \ldots, n$ (in sample), can be obtained by replacing $\gamma$ by its CMLE, $\widehat{\gamma}$, and $r_{t}$ by $g\left(y_{t}\right)-g\left(\widehat{\mu}_{t}\right)$ in the Equation (1). The $h$ step ahead forecast, $h=1,2, \ldots$, can be computed as

$$
\begin{array}{r}
\widehat{\mu}_{n+h}=g^{-1}\left(\widehat{\beta}+\sum_{i=1}^{p} \widehat{\phi}_{i}\left[g\left(y_{n+h-i}\right)\right]-\sum_{j=1}^{q} \widehat{\theta}_{j}\left[r_{n+h-j}\right]+\sum_{I=1}^{P} \widehat{\Phi}_{I}\left[g\left(y_{n+h-I S}\right)\right]-\sum_{J=1}^{Q} \widehat{\Theta}_{J}\left[r_{n+h-J S}\right]\right. \\
\left.-\sum_{i=1}^{p} \sum_{I=1}^{P} \widehat{\phi}_{i} \widehat{\Phi}_{I}\left[g\left(y_{n+h-(i+I S)}\right)\right]+\sum_{j=1}^{q} \sum_{J=1}^{Q} \widehat{\theta}_{j} \widehat{\Theta}_{J}\left[r_{n+h-(j+J S)}\right]\right),
\end{array}
$$

where

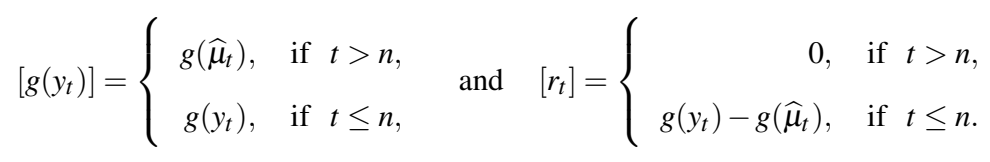

\section{Numerical evaluation}

We performed Monte Carlo simulations to evaluate the finite sample performance of the CMLE and also the accuracy of white noise testing inference. We used $R=10,000$ Monte Carlo replications and the following sample sizes: $n=50,100,200,500$. In each Monte Carlo replication we generate a vector of $n$ occurrences of the variable $y_{t}$ from the $\beta$ SARMA model given in 11 with logit link. The parameter values are presented in Table 1 along with the numerical results. We report the mean of all estimates, and also estimates of the bias, estimated relative bias (RB), standard deviation (SD), and mean square error (MSE). All conditional log-likelihood maximizations were carried out using the BFGS quasi-Newton method with analytical first derivatives. Starting values for the autoregressive parameters 


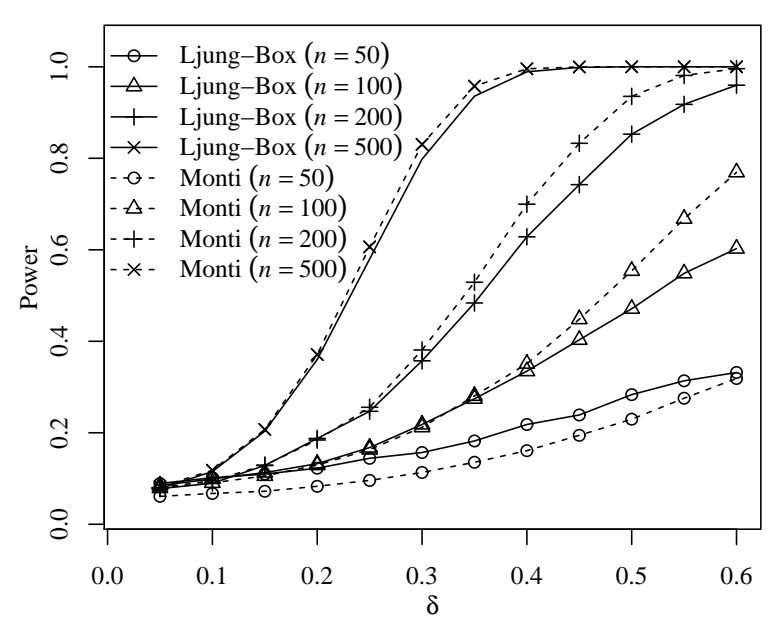

(a) Scenario 1

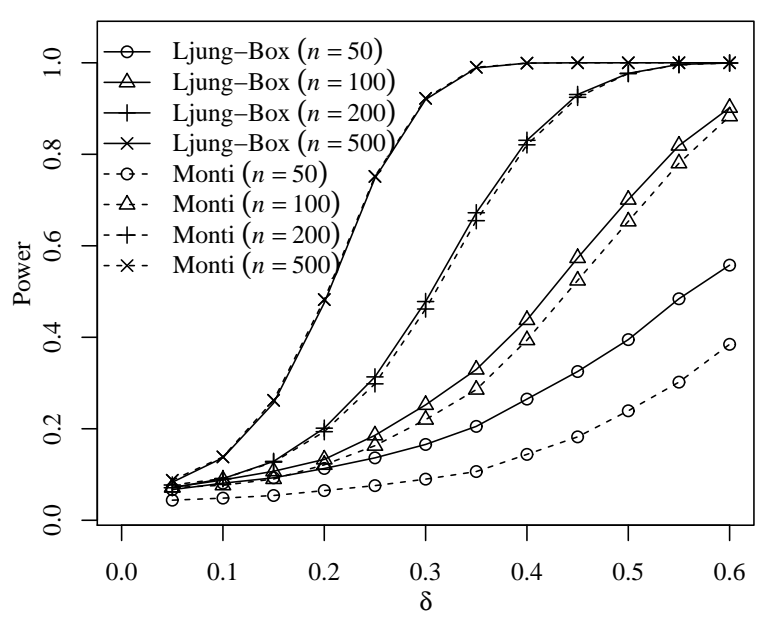

(b) Scenario 2

Figure 2: Nonnull rejection rates (powers) of Ljung-Box and Monti tests; $\alpha=5 \%$.

were obtained by regressing $g\left(y_{t}\right)$ on $g\left(y_{t-1}\right), \ldots, g\left(y_{t-p}\right), g\left(y_{t-(p+1)}\right), \ldots, g\left(y_{t-(p+P)}\right)$, and the all moving average parameters were set equal to zero at the beginning of the conditional log-likelihood maximizations. The simulations were performed using the R statistical computing environment [84].

The results in Table 1 show that the estimator of the autoregressive parameter $\phi_{1}$ is nearly unbiased. Similar to what happens in beta regressions [85], the precision parameter estimator displays some small sample bias. The remaining estimators display substantial bias when the sample size is small. Such biases become smaller as the sample size grows.

We also estimated the null rejection rates (sizes) of the Ljung-Box and Monti-type tests presented in Section 4.4 based on the standardized weighted residual $\left(\hat{r}_{t}^{w}\right)$. The simulation setup was the same as in the previous set of simulations. The tests nominal levels are $10 \%, 5 \%$ and $1 \%$ and we used $b=\max (10,2 S)$, with $S=12$. The results are displayed in Table 2 They show that the Monti test typically outperforms the Ljung-Box test. We have also used Monte Carlo simulation to evaluate the tests nonnull rejection rates (powers). Figure 2 presents such rates for two scenarios:

Scenario 1: Data generation was carried out from $\beta \operatorname{SARMA}(1,1)(1,1)$ with parameters equal to $\beta=-1.00, \phi_{1}=$ $-0.50, \Phi_{1}=0.30, \theta_{1}=\delta, \Theta_{1}=-0.35$, and $\varphi=120$, and fitted model was $\beta \operatorname{SARMA}(1,0)(1,1)$.

Scenario 2: Data generation was carried out from $\beta \operatorname{SARMA}(2,1)(1,1)$ with parameters equal to $\beta=-1.00, \phi_{1}=$ $-0.50, \phi_{2}=-\delta, \Phi_{1}=0.30, \theta_{1}=0.40, \Theta_{1}=-0.35$, and $\varphi=120$, and fitted model was $\beta \operatorname{SARMA}(1,1)(1,1)$. In both scenarios, $\delta=0.05,0.10, \ldots, 0.55,0.60$. As expected, the tests become more powerful as $\delta$ moves away from zero and as the sample size increases. In small samples the Ljung-Box test is more powerful than the Monti test; in large samples, however, they are nearly equally powerful. Overall, both tests seem to work well. 
Table 1: Simulation results on point estimation, $\beta \operatorname{SARMA}(1,1) \times(1,1)_{12}$

\begin{tabular}{|c|c|c|c|c|c|c|}
\hline Parameters & $\begin{array}{r}\beta \\
-1.000\end{array}$ & $\begin{array}{r}\phi_{1} \\
-0.500\end{array}$ & $\begin{array}{r}\Phi_{1} \\
0.300\end{array}$ & $\begin{array}{r}\theta_{1} \\
0.400\end{array}$ & $\begin{array}{r}\Theta_{1} \\
-0.350\end{array}$ & $\begin{array}{r}\varphi \\
120.000\end{array}$ \\
\hline \multicolumn{7}{|c|}{$n=50$} \\
\hline Mean & -0.7494 & -0.4725 & 0.4626 & 0.4415 & -0.1233 & 99.6751 \\
\hline Bias & -0.2506 & -0.0275 & -0.1626 & -0.0415 & -0.2267 & 20.3249 \\
\hline $\mathrm{RB}(\%)$ & 25.0592 & 5.5057 & -54.1886 & -10.3711 & 64.7802 & 16.9374 \\
\hline SD & 0.3296 & 0.2065 & 0.2189 & 0.2542 & 0.3357 & 29.6409 \\
\hline MSE & 0.1714 & 0.0434 & 0.0744 & 0.0664 & 0.1641 & 1291.6873 \\
\hline \multicolumn{7}{|c|}{$n=100$} \\
\hline Mean & -0.8698 & -0.4807 & 0.3816 & 0.4181 & -0.2464 & 96.9399 \\
\hline Bias & -0.1302 & -0.0193 & -0.0816 & -0.0181 & -0.1036 & 23.0601 \\
\hline $\mathrm{RB}(\%)$ & 13.0189 & 3.8569 & -27.1955 & -4.5257 & 29.6053 & 19.2167 \\
\hline SD & 0.2425 & 0.1343 & 0.1604 & 0.1487 & 0.1940 & 18.2091 \\
\hline MSE & 0.0758 & 0.0184 & 0.0324 & 0.0224 & 0.0484 & 863.3406 \\
\hline \multicolumn{7}{|c|}{$n=200$} \\
\hline Mean & -0.9363 & -0.4855 & 0.3371 & 0.4132 & -0.3018 & 105.2458 \\
\hline Bias & -0.0637 & -0.0145 & -0.0371 & -0.0132 & -0.0482 & 14.7542 \\
\hline $\mathrm{RB}(\%)$ & 6.3663 & 2.8974 & -12.3528 & -3.3068 & 13.7660 & 12.2952 \\
\hline SD & 0.1830 & 0.0928 & 0.1238 & 0.0994 & 0.1360 & 13.7747 \\
\hline MSE & 0.0375 & 0.0088 & 0.0167 & 0.0101 & 0.0208 & 407.4279 \\
\hline \multicolumn{7}{|c|}{$n=500$} \\
\hline Mean & -0.9842 & -0.4947 & 0.308 & 0.404 & -0.3385 & 111.9313 \\
\hline Bias & -0.0158 & -0.0053 & -0.008 & -0.004 & -0.0115 & 8.0687 \\
\hline $\mathrm{RB}(\%)$ & 1.5808 & 1.0617 & -2.6824 & -0.9898 & 3.2979 & 6.7240 \\
\hline SD & 0.1205 & 0.0563 & 0.0805 & 0.0606 & 0.0825 & 9.4855 \\
\hline MSE & 0.0148 & 0.0032 & 0.0066 & 0.0037 & 0.0069 & 155.0802 \\
\hline
\end{tabular}

Table 2: Null rejection rates of the Ljung-Box and Monti tests

\begin{tabular}{lrrrr}
\hline & $n=50$ & $n=100$ & $n=200$ & $n=500$ \\
\hline \multicolumn{5}{c}{$\alpha=10 \%$} \\
\hline Ljung-Box & 10.77 & 12.56 & 11.27 & 11.97 \\
Monti & 9.55 & 13.59 & 12.53 & 12.49 \\
\hline \multicolumn{5}{c}{$\alpha=5 \%$} \\
Ljung-Box & 6.29 & 7.24 & 6.10 & 6.15 \\
Monti & 3.94 & 6.98 & 6.63 & 6.39 \\
\hline \multicolumn{5}{c}{$\alpha=1 \%$} \\
Ljung-Box & 1.97 & 2.36 & 1.58 & 1.52 \\
Monti & 0.53 & 1.26 & 1.51 & 1.46 \\
\hline
\end{tabular}




\section{Empirical application}

We shall now model data on air relative humidity (RH) in Santa Maria, RS, Brazil. The data consist of monthly averages from January 2003 through October 2017 and were obtained from Banco de Dados Meteorológicos para Ensino e Pesquisa do Instituto Nacional de Meteorologia (INMET) [86]. The last ten observations were removed from the data and used for forecasting evaluation. Figure 3 contains the time series data plot and also plots of the sample autocorrelation (ACF) and sample partial autocorrelation (PACF) functions. The sinusoidal patterns in both correlograms are indicative of seasonal dynamics.

The importance of modeling and forecasting environmental variables such as RH is largely discussed in literature [87, 42, 43, 44]. In particular, humidity seasonality has been linked to several infectious diseases [87]. It is thus important that seasonal fluctuations be properly modeled and that accurate forecasts are available to policymakers.

We selected the $\beta \operatorname{SARMA}(1,0) \times(1,1)_{12}$ model for the data at hand. Parameter estimates, standard errors, $z$ statistics and their $p$-values are given in Table 3 Some diagnostic measures and additional test statistics are also included in the table. Notice that the seasonality test $p$-value is quite small, which is suggestive of strong seasonal dynamics. In addition, the Ljung-Box and Monti tests based on the standardized weighted residual do not reject the null hypothesis that the first $b$ residual autocorrelations are equal to zero. Hence, the model seems to be correctly specified.

Figure 4 contains six plots, namely: (a) observed versus fitted values, (b) index plot of the standardized residuals, (c) residual sample autocorrelation function, (d) residual sample partial autocorrelation function, (e) residuals QQ (quantile-quantile) plot, and (f) residual density estimate obtained using a Gaussian kernel, which is plotted alongside the standard normal density. All plots and tests indicate that the fitted model can be safely used for out-of-sample forecasting. In Figure 5(a) we plot the data (solid line) together with in-sample predictions (dashed line).

We forecasted the next ten (out-of-sample) observations using the $\beta \operatorname{SARMA}(1,0) \times(1,1)$ model, the $\operatorname{SARIMA}(1,0,0) \times(1,0,1)$ and also exponential smoothing state space models (ETS) [88]. (Recall that such observations were removed from the data at the outset.) The SARIMA and ETS forecasts were produced using the forecast R package [89]. The three sets of out-of-sample forecasts are presented in Figure 5(b)] Table 4 presents the mean square error (MSE) and the mean absolute percentage error (MAPE) computed for each methodology. It is noteworthy that the $\beta$ SARMA forecasts are the most accurate according to both criteria. 


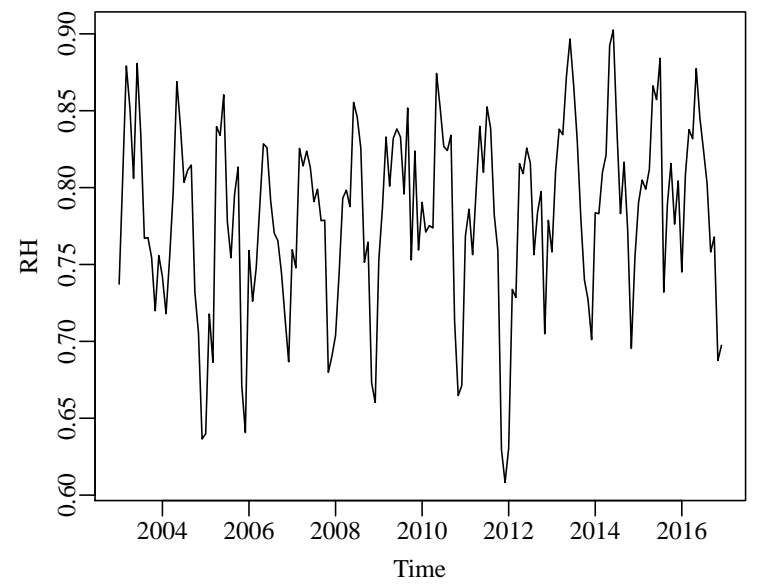

(a) RH series

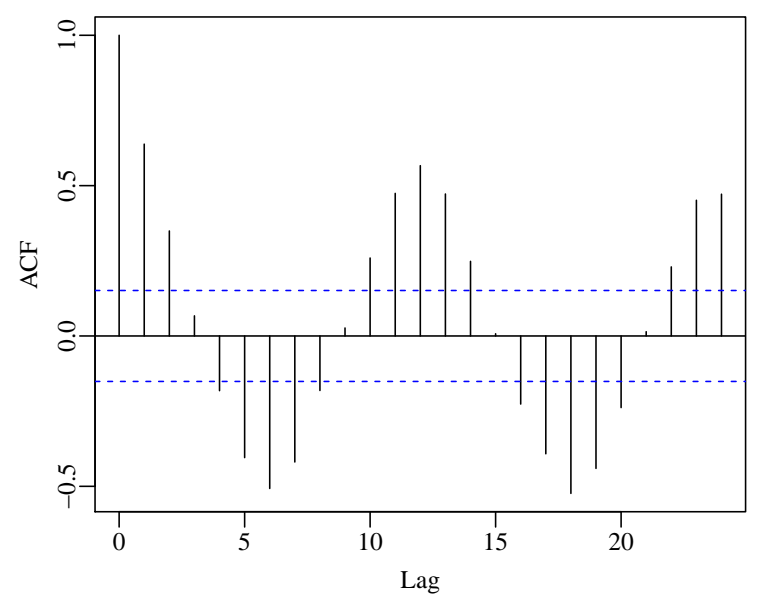

(c) $\mathrm{ACF}$

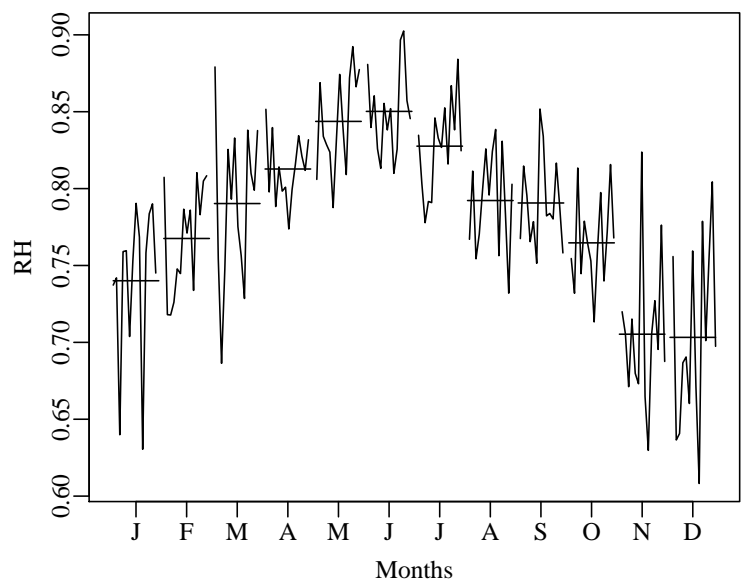

(b) Seasonality

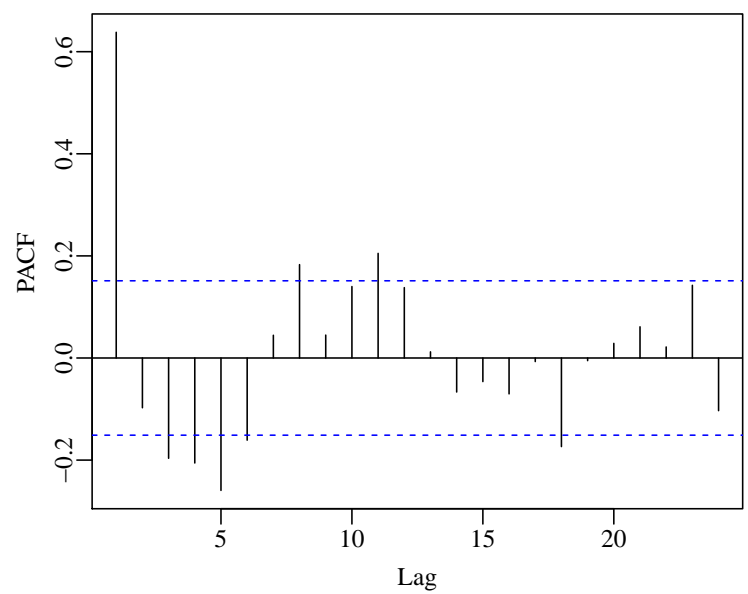

(d) PACF

Figure 3: Time series of relative humidity of air in Santa Maria, Brazil. 


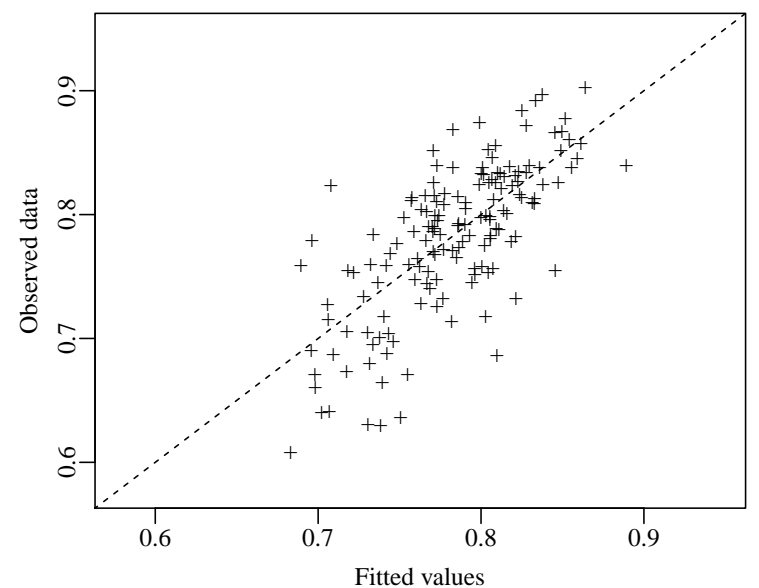

(a) Observed versus fitted

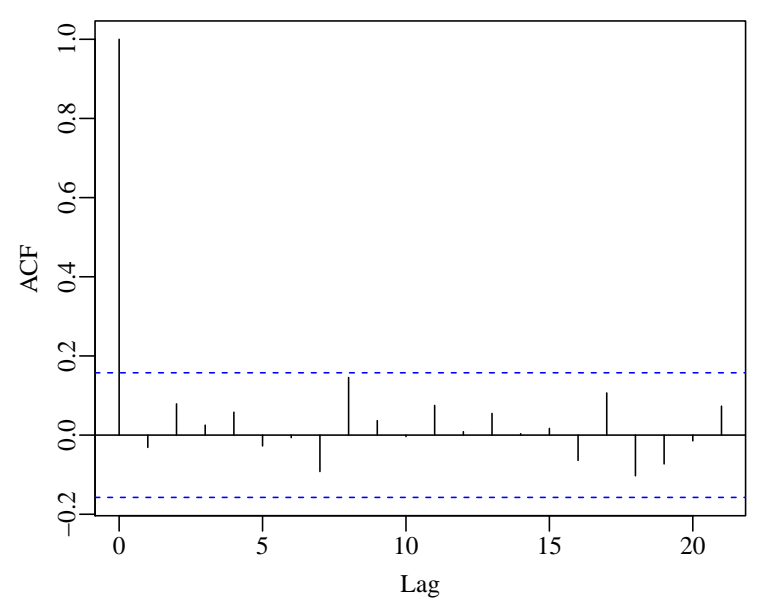

(c) Residual ACF

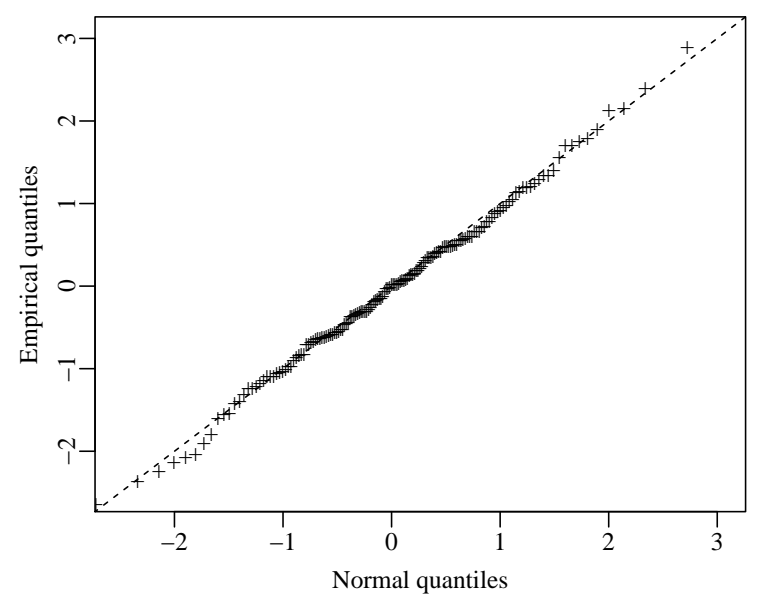

(e) QQ-plot

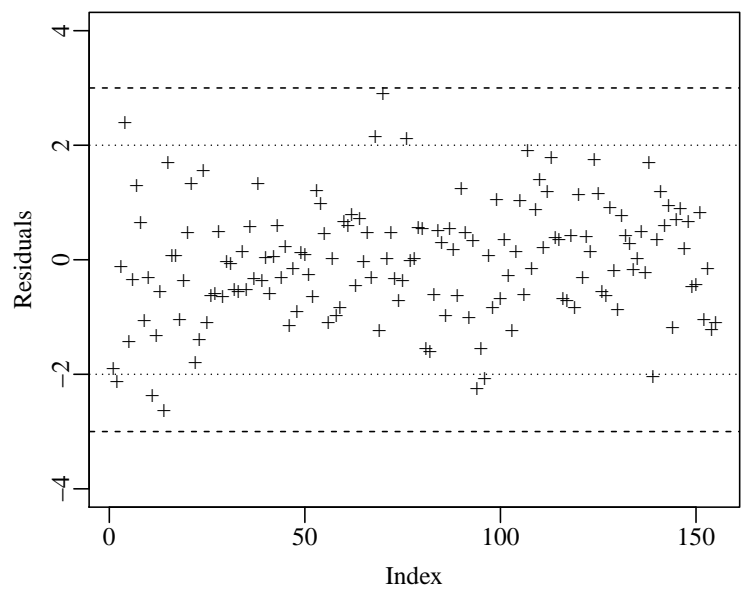

(b) Standardized weighted residual

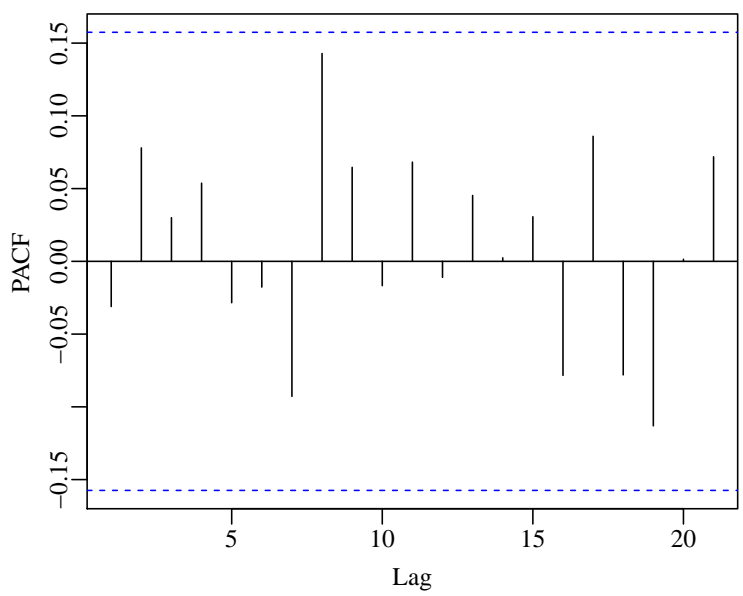

(d) Residual PACF

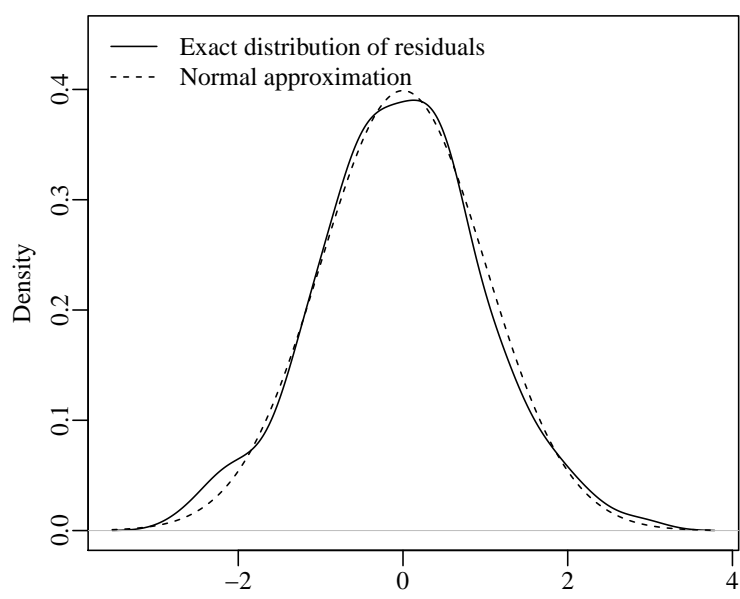

(f) Residual distribution

Figure 4: Diagnostic plots based on standardized weighted residuals $\left(r_{t}^{w}\right)$. 
Table 3: Fitted $\beta \operatorname{SARMA}(1,0) \times(1,1)_{12}$ model

\begin{tabular}{lcccc}
\hline & estimate & std. error & z stat. & $p$-value \\
\hline$\beta$ & 0.1057 & 0.0365 & 2.8973 & 0.0038 \\
$\phi_{1}$ & 0.3834 & 0.0437 & 8.7657 & $<0.0001$ \\
$\Phi_{1}$ & 0.8615 & 0.0586 & 14.7071 & $<0.0001$ \\
$\Theta_{1}$ & 0.5668 & 0.0709 & 7.9892 & $<0.0001$ \\
$\varphi$ & 98.3114 & 11.1297 & 8.8332 & $<0.0001$ \\
\hline \multicolumn{5}{c}{ Log-likelihood $=298.9695$} \\
Deviance $=153.5969$ \\
MAIC $=-585.9390$ & MSIC $=-567.1952$
\end{tabular}

Seasonality test: $W=265.2603$ ( $p$-value $<0.0001)$

Ljung-Box test: $Q_{1}=23.555(p$-value $=0.2624)$

Monti test: $Q_{2}=22.728(p$-value $=0.3023)$

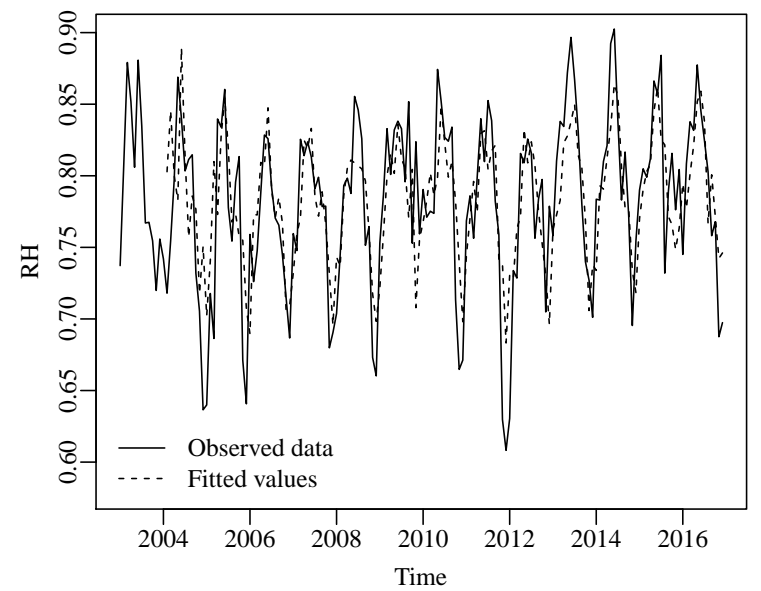

(a) Fitted $\beta \operatorname{SARMA}(1,0) \times(1,1)$

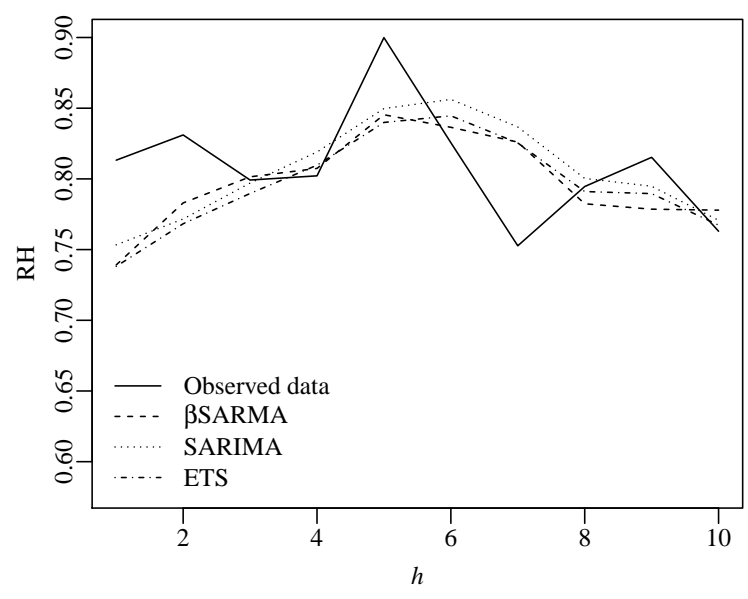

(b) Observed and predicted values, January through October 2017

Figure 5: Observed, fitted and predicted values of RH in Santa Maria, Brazil.

Table 4: MSE and MAPE for predicted values from different models

\begin{tabular}{lcc}
\hline Model & MSE & MAPE \\
\hline$\beta$ SARMA & 0.00180 & 0.04094 \\
SARIMA & 0.00197 & 0.04172 \\
ETS & 0.00184 & 0.04158 \\
\hline
\end{tabular}




\section{Conclusions}

Oftentimes practitioners need to model and predict the future behavior of times series that assume values in the standard unit interval. The interest may lie, for example, in modeling the behavior of a rate (e.g., unemployment rate) or of a proportion over time. Such time series dynamics may be impacted by seasonal fluctuations. In this paper, we introduced the class of seasonal $\beta$ ARMA models, $\beta$ SARMA. It generalizes the class of $\beta$ ARMA processes and can be used to model and predict time series that assume values in the standard unit interval and are subject to seasonal fluctuations. We showed that parameter estimation can be carried out by conditional maximum likelihood. We derived closed-form expressions for the score vector and for the conditional information matrix. Interval estimation, hypothesis testing inference and model selection were also covered. We presented three different residuals that can be used to assess goodness-of-fit and two white noise noise tests that can be applied to the residuals computed from the fitted model. We also provided Monte Carlo evidence on the finite sample accuracy of point estimation and of two white noise tests. An empirical application was presented and discussed.

\section{Acknowledgments}

We thank an anonymous referee for comments and suggestions. We also gratefully acknowledge partial financial support from CNPq/Brazil.

\section{References}

[1] M.-D. Chuang and G.-H. Yu, "Order series method for forecasting non-Gaussian time series," Journal of Forecasting, vol. 26, no. 4, pp. 239-250, 2007.

[2] G. Box, G. M. Jenkins, and G. Reinsel, Time series analysis: forecasting and control. Hardcover, John Wiley \& Sons, June 2008.

[3] M. L. Tiku, W.-K. Wong, D. C. Vaughan, and G. Bian, "Time series models in non-normal situations: Symmetric innovations," Journal of Time Series Analysis, vol. 21, no. 5, pp. 571-596, 2000.

[4] T. Zheng, H. Xiao, and R. Chen, "Generalized ARMA models with martingale difference errors," Journal of Econometrics, vol. 189, no. 2, pp. 492-506, 2015.

[5] E. McKenzie, "Some models for discrete variate time series," Journal of the American Water Resources Association, vol. 21, no. 4, pp. 645-650, 1985.

[6] M. A. Al-Osh and A. A. Alzaid, "First-order integer-valued autoregressive (INAR(1)) process," Journal of Time Series Analysis, vol. 8, no. 3, pp. 261-275, 1987. 
[7] M. M. Ristić and A. S. Nastić, "A mixed INAR(p) model," Journal of Time Series Analysis, vol. 33, no. 6, pp. 903-915, 2012.

[8] S. L. Zeger and B. Qaqish, "Markov regression models for time series: A quasi-likelihood approach," Biometrics, vol. 44, no. 4, pp. pp. 1019-1031, 1988.

[9] W. K. Li and A. I. McLeod, “ARMA modelling with non-gaussian innovations,” Journal of Time Series Analysis, vol. 9, no. 2, pp. 155-168, 1988.

[10] G. J. Janacek and A. L. Swift, “A class of models for non-normal time series,” Journal of Time Series Analysis, vol. 11, no. 1, pp. 19-31, 1990.

[11] P. McCullagh and J. Nelder, Generalized linear models. Chapman and Hall, 2nd ed., 1989.

[12] W. K. Li, "Testing model adequacy for some markov regression models for time series," Biometrika, vol. 78, no. 1, pp. pp. 83-89, 1991.

[13] W. K. Li, "Time series models based on generalized linear models: Some further results," Biometrics, vol. 50, no. 2, pp. pp. 506-511, 1994.

[14] M. A. Benjamin, R. A. Rigby, and D. M. Stasinopoulos, "Generalized autoregressive moving average models," Journal of the American Statistical Association, vol. 98, no. 461, pp. 214-223, 2003.

[15] K. Fokianos and B. Kedem, "Partial likelihood inference for time series following generalized linear models," Journal of Time Series Analysis, vol. 25, no. 2, pp. 173-197, 2004.

[16] C. H. Sim, "Modelling non-normal first-order autoregressive time series," Journal of Forecasting, vol. 13, no. 4, pp. 369-381, 1994.

[17] A. L. Swift, "Modeling and forecasting time series with a general non-normal distribution," Journal of Forecasting, vol. 14, no. 1, pp. 45-66, 1995.

[18] A. D. Akkaya and M. L. Tiku, “Time series AR(1) model for short-tailed distributions,” Statistics, vol. 39, no. 2, pp. 117-132, 2005.

[19] R. C. Jung, M. Kukuk, and R. Liesenfeld, "Time series of count data: modeling, estimation and diagnostics," Computational Statistics \& Data Analysis, vol. 51, no. 4, pp. 2350-2364, 2006.

[20] P. Bondon, "Estimation of autoregressive models with epsilon-skew-normal innovations," Journal of Multivariate Analysis, vol. 100, no. 8, pp. 1761-1776, 2009.

[21] B. Kedem and K. Fokianos, Regression models for time series analysis. John Wiley \& Sons, 2002.

[22] P. Kumaraswamy, “A generalized probability density function for double-bounded random processes,” Journal of Hydrology, vol. 46, pp. 79-88, 1980. 
[23] G. K. Grunwald, A. E. Raftery, and P. Guttorp, “Time series of continuous proportions," Journal of the Royal Statistical Society. Series B, vol. 55, no. 1, pp. 103-116, 1993.

[24] S. L. P. Ferrari and F. Cribari-Neto, "Beta regression for modelling rates and proportions," Journal of Applied Statistics, vol. 31, no. 7, pp. 799-815, 2004.

[25] N. Johnson and S. K. . N. Balakrishnan, “Continuous univariate distributions,” Wiley, no. 2nd, 1995.

[26] R. Kieschnick and B. D. McCullough, "Regression analysis of variates observed on $(0,1)$ : percentages, proportions and fractions," Statistical Modelling, vol. 3, no. 3, pp. 193-213, 2003.

[27] T. F. Melo, K. L. Vasconcellos, and A. J. Lemonte, "Some restriction tests in a new class of regression models for proportions," Computational Statistics \& Data Analysis, vol. 53, no. 12, pp. 3972 - 3979, 2009.

[28] A. K. Gupta, International Encyclopedia of Statistical Science, ch. Beta Distribution, pp. 144-145. Berlin, Heidelberg: Springer Berlin Heidelberg, 2011.

[29] A. Guolo and C. Varin, "Beta regression for time series analysis of bounded data, with application to canada google flu trends," The Annals of Applied Statistics, vol. 8, pp. 74-88, 032014.

[30] A. V. Rocha and F. Cribari-Neto, "Beta autoregressive moving average models," Test, vol. 18, no. 3, pp. 529_ 545, 2009.

[31] W. H. Bonat, P. J. Ribeiro-Jr, and S. E. Shimakura, "Bayesian analysis for a class of beta mixed models," Chilean Journal of Statistics, vol. 6, no. 1, pp. 3-13, 2015.

[32] R. Casarin, L. Dalla Valle, and F. Leisen, "Bayesian model selection for beta autoregressive processes," Bayesian Analysis, vol. 7, pp. 385-410, 062012.

[33] C. Q. Silva, H. S. Migon, and L. T. Correia, "Dynamic bayesian beta models," Computational Statistics \& Data Analysis, vol. 55, no. 6, pp. 2074-2089, 2011.

[34] G. Ferreira, J. I. Figueroa-Zúñiga, and M. de Castro, "Partially linear beta regression model with autoregressive errors," Test, vol. 24, no. 4, pp. 752-775, 2015.

[35] R. Lund and I. V. Basawa, "Recursive prediction and likelihood evaluation for periodic ARMA models," Journal of Time Series Analysis, vol. 21, no. 1, pp. 75-93, 2000.

[36] I. Basawa, R. Lund, and Q. Shao, "First-order seasonal autoregressive processes with periodically varying parameters," Statistics \& Probability Letters, vol. 67, no. 4, pp. 299 -306, 2004.

[37] M. Monteiro, M. G. Scotto, and I. Pereira, "Integer-valued autoregressive processes with periodic structure," Journal of Statistical Planning and Inference, vol. 140, no. 6, pp. 1529 -1541, 2010.

[38] D. Moriña, P. Puig, J. Ríos, A. Vilella, and A. Trilla, "A statistical model for hospital admissions caused by seasonal diseases," Statistics in Medicine, vol. 30, no. 26, pp. 3125-3136, 2011. 
[39] O. J. T. Briët, P. H. Amerasinghe, and P. Vounatsou, "Generalized seasonal autoregressive integrated moving average models for count data with application to malaria time series with low case numbers," PLoS ONE, vol. 8, p. e65761, 062013.

[40] M. Bourguignon, K. L. Vasconcellos, V. A. Reisen, and M. de Castrorton Ispány, "A poisson INAR(1) process with a seasonal structure," Journal of Statistical Computation and Simulation, vol. 86, no. 2, pp. 373-387, 2016.

[41] D. Mitchell, P. Brockett, R. Mendoza-Arriaga, and K. Muthuraman, "Modeling and forecasting mortality rates," Insurance: Mathematics and Economics, vol. 52, no. 2, pp. 275 -285, 2013.

[42] K. Dietz, "The incidence of infectious diseases under the influence of seasonal fluctuations," in Mathematical Models in Medicine (J. Berger, W. Bühler, R. Repges, and P. Tautu, eds.), vol. 11 of Lecture Notes in Biomathematics, pp. 1-15, Springer Berlin Heidelberg, 1976.

[43] D. S.F., "Seasonal variation in host susceptibility and cycles of certain infectious diseases," Emerging Infectious Diseases, vol. 7, no. 3, pp. 369-374, 2001.

[44] N. C. Grassly and C. Fraser, "Seasonal infectious disease epidemiology," Proceedings of the Royal Society of London B: Biological Sciences, vol. 273, no. 1600, pp. 2541-2550, 2006.

[45] K. S. Emara and A. E. Shalaby, "Seasonal variation of fixed and volatile oil percentage of four eucalyptus spp. related to lamina anatomy," African Journal of Plant Science, vol. 5, no. 6, pp. 353-359, 2011.

[46] R. S. Mullen, Modeling the temporal and spatial variability of solar radiation. PhD thesis, Montana State University, 2012. Doctor in Ecology and Environmental Sciences.

[47] V. Sundar and K. Subbiah, "Application of double bounded probability density function for analysis of ocean waves," Ocean Engineering, vol. 16, no. 2, pp. 193-200, 1989.

[48] S. Fletcher and K. Ponnambalam, "Estimation of reservoir yield and storage distribution using moments analysis," Journal of Hydrology, vol. 182, no. 1-4, pp. 259-275, 1996.

[49] A. Ganji, K. Ponnambalam, D. Khalili, and M. Karamouz, "Grain yield reliability analysis with crop water demand uncertainty," Stochastic Environmental Research and Risk Assessment, vol. 20, no. 4, pp. 259-277, 2006.

[50] B. A. Andersen, "Asymptotic properties of conditional maximum-likelihood estimators," Journal of the Royal Statistical Society. Series B, vol. 32, no. 1, pp. 283-301, 1970.

[51] M. A. Benjamin, R. A. Rigby, and D. M. Stasinopoulos, "Fitting non-Gaussian time series models," COMPSTAT Proceedings in Computational Statistics, vol. Heidelburg: Physica-Verlag, pp. 191-196, 1998.

[52] A. V. Rocha and F. Cribari-Neto, "Erratum to: Beta autoregressive moving average models," Test, vol. 26, no. 2, pp. 451-459, 2017. 
[53] J. Nocedal and S. J. Wright, Numerical optimization. Springer, 1999.

[54] W. Press, S. Teukolsky, W. Vetterling, and B. Flannery, Numerical recipes in C: The art of scientific computing. Cambridge University Press, 2nd edition ed., 1992.

[55] D. R. Cox and N. Reid, "Parameter orthogonality and approximate conditional inference," Journal of the Royal Statistical Society. Series B, vol. 49, no. 1, pp. 1-39, 1987.

[56] Y. Pawitan, In All Likelihood: Statistical Modelling and Inference Using Likelihood. Oxford Science publications, 2001.

[57] A. C. Davison and D. V. Hinkley, Bootstrap Methods and Their Application. Cambridge University Press, 1997.

[58] J. Neyman and E. S. Pearson, "On the use and interpretation of certain test criteria for purposes of statistical inference," Biometrika, vol. 20A, no. 1/2, pp. 175-240, 1928.

[59] C. Rao, "Large sample tests of statistical hypotheses concerning several parameters with applications to problems of estimation," Mathematical Proceedings of the Cambridge Philosophical Society, vol. 44, no. 1, pp. 50$57,1948$.

[60] A. Wald, "Tests of statistical hypotheses concerning several parameters when the number of observations is large," Transactions of the American Mathematical Society, vol. 54, pp. 426-482, 1943.

[61] G. R. Terrell, “The gradient statistic,” Computing Science and Statistics, vol. 34, pp. 206-215, 2002.

[62] H. Akaike, "A new look at the statistical model identification," IEEE Transactions on Automatic Control, vol. 19 , no. 6, pp. 716-723, 1974.

[63] W. Pan, "Bootstrapping likelihood for model selection with small samples," Journal of Computational and Graphical Statistics, vol. 8, no. 4, pp. 687-698, 1999.

[64] G. Schwarz, "Estimating the dimension of a model," The Annals of Statistics, vol. 6, no. 2, pp. 461-464, 1978.

[65] E. J. Hannan and B. G. Quinn, “The determination of the order of an autoregression,” Journal of the Royal Statistical Society. Series B, vol. 41, no. 2, pp. 190-195, 1979.

[66] N. Sugiura, "Further analysts of the data by Akaike's information criterion and the finite corrections - further analysts of the data by Akaike's," Communications in Statistics: Theory and Methods, vol. 7, no. 1, pp. 13-26, 1978.

[67] C. M. Hurvich and C.-L. Tsai, "Regression and time series model selection in small samples," Biometrika, vol. 76, no. 2, pp. 297-307, 1989.

[68] A. McQuarrie, "A small-sample correction for the Schwarz SIC model selection criterion," Statistics \& Probability Letters, vol. 44, no. 1, pp. 79-86, 1999. 
[69] J. Shao, "Bootstrap model selection," Journal of the American Statistical Association, vol. 91, no. 434, pp. 655665, 1996.

[70] J. Cavanaugh, "Unifying the derivations for the Akaike and corrected Akaike information criteria," Statistics \& Probability Letters, vol. 33, no. 2, pp. 201-208, 1997.

[71] M. Ishiguro, Y. Sakamoto, and G. Kitagawa, "Bootstrapping log likelihood and EIC, an extension of AIC," Annals of the Institute of Statistical Mathematics, vol. 49, no. 3, pp. 411-434, 1997.

[72] J. E. Cavanaugh and R. H. Shumway, "A bootstrap variant of AIC for state-space model selection," Statistica Sinica, vol. 7, pp. 473-496, 1997.

[73] R. Shibata, "Bootstrap estimate of Kullback-Leibler information for model selection," Statistica Sinica, vol. 7, pp. 375-394, 1997.

[74] A.-K. Seghouane, “Asymptotic bootstrap corrections of AIC for linear regression models," Signal Processing, vol. 90, pp. 217-224, 2010.

[75] F. Bayer and F. Cribari-Neto, "Bootstrap-based model selection criteria for beta regressions," Test, vol. 24, no. 4, pp. 776-795, 2015.

[76] R. H. Myers, D. C. Montgomery, G. G. Vining, and T. J. Robinson, Generalized Linear Models with Applications in Engineering and the Sciences. Wiley, 2 ed., 2010.

[77] J. A. Mauricio, "Computing and using residuals in time series models," Computational Statistics \& Data Analysis, vol. 52, no. 3, pp. 1746-1763, 2008.

[78] P. L. Espinheira, S. L. P. Ferrari, and F. Cribari-Neto, "Influence diagnostics in beta regression,” Computational Statistics \& Data Analysis, vol. 52, pp. 4417-4431, 2008.

[79] P. Espinheira, S. L. P. Ferrari, and F. Cribari-Neto, “On beta regression residuals," Journal of Applied Statistics, vol. 35, pp. 407-419, 2008.

[80] R. L. Anderson, "Distribution of the serial correlation coefficient," The Annals of Mathematical Statistics, vol. 13, no. 1, pp. 1-13, 1942.

[81] G. M. Ljung and G. E. P. Box, "On a measure of lack of fit in time series models," Biometrika, vol. 65, no. 2, pp. pp. 297-303, 1978.

[82] A. C. Monti, “A proposal for a residual autocorrelation test in linear models," Biometrika, vol. 81, no. 4, pp. 776780, 1994.

[83] R. J. Hyndman and G. Athanasopoulos, Forecasting: principles and practice. OTexts, October 2013.

[84] R Development Core Team, R: A Language and Environment for Statistical Computing. R Foundation for Statistical Computing, Vienna, Austria, 2016. ISBN 3-900051-07-0. 
[85] A. B. Simas, W. Barreto-Souza, and A. V. Rocha, "Improved estimators for a general class of beta regression models," Computational Statistics \& Data Analysis, vol. 2, pp. 348-366, 2010.

[86] "INMET." www.inmet.gov.br/projetos/rede/pesquisa, 2018. Banco de Dados Meteorológicos para Ensino e Pesquisa do Instituto Nacional de Meteorologia (INMET), Brazil.

[87] J. D. Tamerius, J. Shaman, W. J. Alonso, K. Bloom-Feshbach, C. K. Uejio, A. Comrie, and C. Viboud, "Environmental predictors of seasonal influenza epidemics across temperate and tropical climates," PLoS Pathogens, vol. 9, p. e1003194, 032013.

[88] A. B. Hyndman, R. J. and Koehler, J. K. Ord, and R. D. Snyder, Forecasting with Exponential Smoothing: The State Space Approach. Springer, 2008.

[89] R. J. Hyndman, forecast: Forecasting functions for time series and linear models, r package version 8.0 ed., 2017. 